\title{
Exploratory Spectroscopy of Magnetic Cataclysmic Variables Candidates and Other Variable Objects
}

\author{
A. S. Oliveira \\ IPÉD, Universidade do Vale do Paraíba, 12244-000, São José dos Campos, SP, Brazil \\ alexandre@univap.br \\ C. V. Rodrigues, D. Cieslinski, F. J. Jablonski \\ Divisão de Astrofísica, Instituto Nacional de Pesquisas Espaciais, 12227-010, São José dos Campos, SP, \\ Brazil \\ K. M. G. Silva \\ Gemini Observatory, Casilla 603, La Serena, Chile \\ L. A. Almeida \\ Instituto de Astronomia, Geofísica e Ciências Atmosféricas, Universidade de São Paulo, 05508-900, São \\ Paulo, SP, Brazil \\ A. Rodríguez-Ardila \\ Laboratório Nacional de Astrofísica LNA/MCTI, 37504-364, Itajubá MG, Brazil \\ and \\ M. S. Palhares \\ IPESD, Universidade do Vale do Paraiba, 12244-000, São José dos Campos, SP, Brazil
}

\begin{abstract}
The increasing number of synoptic surveys made by small robotic telescopes, such as the photometric Catalina Real-Time Transient Survey (CRTS), represents a unique opportunity for the discovery of variable sources and improves the statistical samples of such classes of objects. Our goal is the discovery of magnetic Cataclysmic Variables (mCVs). These are rare objects, which probe interesting accretion scenarios controlled by the white dwarf magnetic field. In particular, improved statistics of mCVs would help to address open questions on their formation and evolution. We performed an optical spectroscopy survey to search for signatures of magnetic accretion in 45 variable objects selected mostly from the CRTS. In this sample we found $32 \mathrm{CVs}$, 22 being $\mathrm{mCV}$ candidates from which 13 are previously unreported as such. If the proposed classifications are confirmed, it would represent an increase of $4 \%$ in the number of known polars and $12 \%$ in the number of known IPs. A fraction of our initial sample was classified as extragalactic sources or other types of variable stars by the inspection of the identification spectra. Despite the inherent complexity in identifying a source as a $\mathrm{mCV}$, variability-based selection followed by spectroscopic snapshot observations has proved to be an efficient strategy for their discoveries, being a relatively inexpensive approach in terms of telescope time.
\end{abstract}

Subject headings: binaries: close — novae, cataclysmic variables — stars: dwarf novae — stars: variables: general — techniques: spectroscopic 


\section{INTRODUCTION}

Cataclysmic Variable stars (CVs) are binary systems consisting of a white dwarf accreting mass from a less massive main sequence or a slightly evolved companion star via Roche lobe overflow (e.g., Warner 1995). For most systems, the secondary is a non-degenerate object, so the mass transfer leads to a decrease in the orbital period and demands a loss of orbital angular momentum that is usually attributed to two complementary mechanisms: magnetic braking of the secondary star, important in longer orbital period systems, and gravitational radiation losses that dominates in shorter period systems, in which the former mechanism is less efficient. See Knigge et al. (2011) for a nice review of CV evolution. Most commonly, the accretion proceeds via an extended accretion disk that surrounds the accreting white dwarf. The disk is usually the dominant source of radiation in the binary system during periods of high accretion. The accretion disk may suffer recurrent episodes of instability known as Dwarf Nova (DN) outbursts, which increase the luminosity of the system by $2-6$ magnitudes in timescales as short as a day.

If the white dwarf has a strong enough magnetic field, however, the transferred ionized material connects to the magnetic field lines and accretes directly to the white dwarf surface, close to the magnetic poles, via an accretion column instead of a disk. These systems are known as magnetic Cataclysmic Variables (mCVs) and can be classified either as polars, whose magnetic fields are intense ( $\mathrm{B} \sim 7-230 \mathrm{MG})$, or as intermediate polars (IPs), in which $\mathrm{B} \sim 1-10$ MG. The distinctive characteristic of the polars is the synchronization of the white-dwarf spin and the orbital period, caused by magnetic locking of the primary and secondary stars (see Cropper 1990, for a review on polars). Matter falling through the accretion column emits cyclotron radiation, which is highly polarized, anisotropic and dominates the optical and infrared emission. Intermediate polars, on the other hand, are not synchronized systems, and the distinct spin, beat and orbital periods manifest in optical and X-rays. IPs usually present an accretion disk, truncated in its inner region by the magnetosphere of the white dwarf.

A large number of CV systems has been dis- covered by surveys based on color selection, such as those identified through SDSS data (e.g., Szkody et al. 2011; York et al. 2000). Surveys based on variability are also prolific in finding new CVs and in the near future, the Large Synoptic Survey Telescope - LSST - is expected to strongly impact the statistics of CVs. One of these variability surveys is the Catalina Real-Time Transient Survey - CRTS (Drake et al. 2009, 2014). CRTS combines three distinct surveys - the Mount Lemmon Survey (MLS) and the Catalina Schmidt Survey (CSS) in the Northern Hemisphere and the Siding Spring Survey (SSS) in the South - covering an area of $30,000 \mathrm{deg}^{2}$ with a typical cadence of two weeks, reaching a depth of 19-21 magnitudes. The CRTS has already found thousands of $\mathrm{CV}$ candidates, which are rapidly announced via their website ${ }^{1}$ and are subject to follow-up investigations (e.g., Coppejans et al. 2014; Drake et al. 2014; Breedt et al. 2014; Thorstensen \& Skinner 2012; Coppejans et al. 2016). As the prime criterion for the CRTS detecting transients is the brightening by 2 or more magnitudes, it turns out to be very effective in discovering non-magnetic $\mathrm{CVs}$, as the follow-up works mentioned above have shown. Other variability surveys that found new CVs are the All Sky Automated Survey (ASAS; Pojmanski 2002), the Palomar Transient Factory (PTF; Margon et al. 2014; Levitan et al. 2015) and the Optical Gravitational Lensing Experiment (OGLE; e.g. Mróz et al. 2013).

The primary goal of this paper is to increase the number of known mCVs systems, which is justified below. These systems are relevant for studying and understanding the physics of magnetically controlled accretion. This phenomenon is widespread in astronomy, from stellar formation processes to accretion on compact objects. But mCVs are relatively simple objects in which the magnetic accretion is observed practically free of other effects allowing a precise description of the process. The short-term variability of $\mathrm{mCVs}$, spanning from minutes to hours, enables us to disentangle the system geometry with a relatively small set of observations. An important aspect of $\mathrm{CV}$ research that can be addressed by an improved statistics of members is the evolution of $\mathrm{mCVs}$, specially the relationship between IPs and

$1_{\text {http }: / / \text { crts.caltech. edu }}$ 
polars (e.g. Ferrario et al. 2015; Pretorius et al. 2013). For instance, it is not known if the surface magnetic field changes along the $\mathrm{mCV}$ evolution. $\mathrm{mCVs}$, IPs in particular, are rare objects and the incompleteness and biases of the present samples are not clear. To determine the frequency distribution of properties as orbital period and primary magnetic field, it is necessary to study individual systems using time-resolved observations and proper modeling (e.g., Costa \& Rodrigues 2009; Silva et al. 2015). Polarimetry is particularly insightful for this, since cyclotron emission is a direct evidence of magnetic fields (e.g., Rodrigues et al. 2006). In particular, our group is building a fast-camera tailored to CVs studies that will perform simultaneous photometry and polarimetry in 4 broad bands (Rodrigues et al. 2012). The 3D modeling of the geometry of the magnetic accretion is an important tool to a realistic representation of $\mathrm{mCVs}$. This kind of analysis can be performed using the Cyclops code, developed by us to study stellar magnetic accretion (Costa \& Rodrigues 2009; Silva et al. 2013).

Here we address the selection of $\mathrm{mCVs}$ candidates for follow-up studies. This paper should be understood as a first step in a broader and ongoing study of mCVs. We performed snapshot spectroscopy of a sample of selected objects. The most promising or peculiar objects will be subject of follow-up time-resolved observations to confirm and/or characterize the systems. Using this strategy, we optimize the use of telescope time.

This paper is organized as follows. Section 2 presents the general rules for the sample selection. Section 3 describes the spectroscopic observations. In Section 4 we present the methods for the time series analysis of the CRTS photometric data. Section 5 describes the criteria applied for the classification of the individual objects of the sample. The spectroscopic results and proposed classification for each object are shown in Section 6. A summary and discussion of the main results are in Section 7.

\section{SAMPLE SELECTION}

In this section we describe the criteria employed to select the final sample of 45 variable objects. We then obtained exploratory spectra (Sec. 3) aiming to identify spectral features that could be signatures of high-ionization mass accretion, typical of mCVs (see Sec. 5).

Our sample is mainly composed by CRTS objects. We also included in the sample $\mathrm{mCV}$ candidates mentioned in the literature that have no published spectroscopic measurements. Previous reports of X-ray detections via $\mathrm{VSNET}^{2}$ and $\mathrm{ATel}^{3}$ alerts were also considered. The classification of $\mathrm{CVs}$ in general and $\mathrm{mCVs}$ in particular can be a difficult task and may demand different observational techniques (as discussed in Sec. 5). This justifies the inclusion of already known mCVs candidates in our sample. Besides, the objects should have suitable apparent magnitudes and positions in the sky for SOAR and OPD observations. No color criterion was applied to define the sample.

The selection of the CRTS objects was done by visual inspection of the light curves with at least one of the following characteristics:

i. Intrinsic flux variability, which may indicate accretion processes. The flux dispersion should be larger than the observational error expected for the mean magnitude;

ii. Transitions between two states of brightness, as seen in polars;

iii. No or few outbursts, in order to minimize dwarf nova detections, although we cannot simply exclude objects with outbursts, since IPs can present such events.

\section{SPECTROSCOPIC OBSERVATIONS AND DATA REDUCTION}

The identification spectra were obtained using the SOAR 4.1-m telescope at Cerro Pachón, Chile, and the Perkin-Elmer 1.6-m telescope at Observatório do Pico dos Dias (OPD-LNA/MCTI), located in Southeast Brazil. The RA ordered list of observed objects is shown in Table 1. The identification of the objects from the CRTS is in the form TTTyymmdd:hhmmss \pm ddmmss, where TTT indicates one of the three dedicated telescopes of the survey - CSS, SSS or MLS - yymmdd is the discovery date of the transient and hhmmss \pm ddmmss

\footnotetext{
${ }^{2}$ http://ooruri.kusastro.kyoto-

u.ac.jp/mailman/listinfo/vsnet-alert

${ }^{3}$ http://www.astronomerstelegram.org/
} 
are the target coordinates. In order to avoid confusion between transients discovered by the same telescope at the same date, and for simplicity, we will abbreviate these IDs as TTThhmm \pm dd.

The SOAR Telescope was operated in service mode and the spectra were obtained using the Goodman High Throughput Spectrograph (Clemens et al. 2004), which employs Volume Phase Holographic (VPH) gratings to maximize throughput, reaching down to the atmospheric cutoff at $3200 \AA$. It is equipped with a Fairchild $4096 \times 4096 \mathrm{CCD}$ with $15 \times 15$ micron pixel $^{-1}(0.15$ " pixel $\left.^{-1}\right)$. The spectrograph was set to operate with the $600 \mathrm{l} \mathrm{mm}^{-1}$ grating, 1.68" slit, and GG 385 blocking filter, yielding a spectral resolution of $7 \AA$ FWHM in the range $4350-7005$ $\AA$. Three exposures of each science target were obtained to remove cosmic rays. $\mathrm{Cu}-\mathrm{He}-\mathrm{Ar}$ lamp exposures were obtained for wavelength calibration, which resulted in typical $0.8 \AA$, or about 45 $\mathrm{km} \mathrm{s}^{-1}$, RMS residuals. The [O I] 5577, 6300 and $6363 \AA$ telluric lines were used to assess the calibration accuracy. The slit was aligned to the parallactic angle to avoid light losses due to the atmospheric differential refraction. Bias images and quartz lamp calibration flats were taken to correct for the detector read-out noise and sensitivity. Spectra of spectrophotometric standards (Hamuy et al. 1992) were used for flux calibration.

The OPD-LNA observations were carried out using a Boller \& Chivens Cassegrain spectrograph on March and September, 2012. A $3001 \mathrm{~mm}^{-1}$ grating, blazed at $6400 \AA$, was used in these two observing runs, providing a spectral coverage in the range $4100-8530 \AA$. The slit aperture was set to $2.50^{\prime \prime}$ in March and $2.00^{\prime \prime}$ in September, giving a spectral resolution of $7 \AA$ and $6 \AA$ (FWHM), respectively. Two different thin, back-illuminated CCD detectors were used: an iKon-L936-BV (March) and an iKon-L936-BRDD (September), both with $2048 \times 2048$ pixels and $13.5 \times 13.5$ micron pixel $^{-1}$. In the March run, a GG 385 blocking filter was used, while in September no filter was necessary. All targets were observed with integration times of 600 or $900 \mathrm{~s}$. Multiple exposures (normally 2 or 3 ) were taken aiming to improve the $\mathrm{S} / \mathrm{N}$ ratio and to minimize effects of cosmic rays in the images. He-Ar comparison lamp exposures were obtained after each target observation for wavelength calibration. For flux calibration, we observed the spectrophotometric standard stars HR 3454 and HR 9087 from Hamuy et al. (1992). In all nights, images of dome flats and bias were also obtained.

The data reduction, spectra extraction and wavelength, extinction and flux calibrations were performed using standard $\operatorname{IRAF}^{4}$ routines.

\section{TIME SERIES ANALYSIS OF CRTS DATA}

We performed time series analyses of the CRTS photometric data for all Galactic objects to search for periodic signals. The sampling of the CRTS light curves has a typical minimum separation between measurements of $\Delta \mathrm{t}=0.01$ days. This means that if the time series was equally spaced with this sampling, the Nyquist frequency would be $\mathrm{F}_{N y}=1 / 2 \Delta \mathrm{t}=50 \mathrm{~d}^{-1}$. The total span of the observations is $\approx 3000 \mathrm{~d}$, which implies (again for equally spaced sampling) a frequency step $\Delta \mathrm{F}$ $=1 / \mathrm{T}=3.3 \times 10^{-4} \mathrm{~d}^{-1}$. The number of frequencies to be examined for coherent signals is $\mathrm{N}=\mathrm{F}_{N y} / \Delta \mathrm{F} \approx 1.5 \times 10^{5}$. We choose to examine a grid of $\mathrm{N}=10^{6}$ frequencies giving some slack to allow for possible effects coming from the nonuniform sampling of the time series data. We are aware that sparse data, as CRTS data, can produce complex structure in the power spectrum due to lateral lobes and aliasing. However, the periods quoted in this article have always a false alarm probability (FAP) less than 0.01 (=1\%) see below how it is quantified.

We used the Discrete Fourier Transform (DFT, Deeming 1975; Lomb 1976), the Lomb-Scargle (LS, Lomb 1976; Scargle 1982) including its Bayesian formalism implementation (BGLS, Mortier et al. 2015), the String-Length (SL, Dworetsky 1983; Clarke 2002) and the Phase Dispersion Minimization (PDM, Stellingwerf 1978) methods for signal search, depending on the characteristics of each light curve. These tools can be classified in two groups, those of the DFT family (DFT, LS, BGLS) based on Fourier transform and those based on phase diagram methods (SL, PDM), which have the advantage of being sensitive even for highly

\footnotetext{
${ }^{4}$ IRAF is distributed by the National Optical Astronomy Observatories, which are operated by the Association of Universities for Research in Astronomy, Inc., under cooperative agreement with the National Science Foundation.
} 
TABLE 1

LIST OF OBSERVED TARGETS.

\begin{tabular}{|c|c|c|c|c|c|c|c|}
\hline Object name & Abbreviation & RA (J2000) & Dec (J2000) & Date obs. & Telesc. & Exp. time (s) & Type $^{a}$ \\
\hline CSS091009:010412-031341 & CSS0104-03 & 01:04:12 & $-03: 13: 41$ & 2012 Aug 25 & SOAR & 3600 & $\mathrm{D} / \mathrm{IP}$ \\
\hline CSS091215:021311+002153 & CSS0213+00 & $02: 13: 11$ & $+00: 21: 53$ & 2012 Sep 09 & SOAR & 8100 & $\mathrm{E}$ \\
\hline MLS110213:022733+130617 & MLS0227+13 & $02: 27: 33$ & $+13: 06: 17$ & 2012 Sep 09 & SOAR & 3600 & $\mathrm{P}$ \\
\hline CSS071206:031525-014051 & CSS0315-01 & $03: 15: 25$ & $-01: 40: 51$ & 2012 Nov 13 & SOAR & 8100 & $\mathrm{E}$ \\
\hline CSS090922:032603+252534 & $\mathrm{CSS} 0326+25$ & $03: 26: 03$ & $+25: 25: 34$ & 2012 Nov 20 & SOAR & 3600 & $\mathrm{D}$ \\
\hline CSS091109:035759+102943 & CSS0357+10 & $03: 57: 59$ & $+10: 29: 43$ & 2012 Nov 12 & SOAR & 8100 & $\mathrm{P}$ \\
\hline MLS101203:045625+182634 & MLS0456+18 & $04: 56: 25$ & $+18: 26: 34$ & 2012 Nov 13 & SOAR & 8100 & $\mathrm{P}$ \\
\hline XMMSL1 J063045.9-603110 & XMM0630-60 & $06: 30: 45$ & $-60: 31: 13$ & 2012 Nov 12 & SOAR & 8100 & $\mathrm{~N}$ \\
\hline MLS101226:072033+172437 & MLS0720+17 & $07: 20: 33$ & $+17: 24: 37$ & 2012 Nov 12 & SOAR & 1440 & $\mathrm{P}$ \\
\hline 1RXS J072103.3-055854 & 1RXS0721-05 & $07: 21: 03$ & $-05: 59: 20$ & 2012 May 01 & SOAR & 1440 & $\mathrm{HA}$ \\
\hline MLS120127:085402+133633 & MLS0854+13 & 08:54:02 & $+13: 36: 33$ & 2012 Dec 15 & SOAR & 8100 & $\mathrm{P}$ \\
\hline 1RXS J100211.4-192534 & 1RXS1002-19 & $10: 02: 11$ & $-19: 25: 36$ & 2012 May 30 & SOAR & 360 & $\mathrm{P}$ \\
\hline CSS120324:101217-182411 & CSS1012-18 & $10: 12: 17$ & $-18: 24: 11$ & 2012 May 30 & SOAR & 3600 & $\mathrm{D} / \mathrm{IP}$ \\
\hline SSS110504:101240-325831 & SSS1012-32 & $10: 12: 40$ & $-32: 58: 31$ & 2012 Apr 21 & SOAR & 3600 & $\mathrm{D}$ \\
\hline CSS110225:112749-054234 & CSS1127-05 & $11: 27: 49$ & $-05: 42: 34$ & 2012 Mar 18 & SOAR & 1440 & $\mathrm{P}$ \\
\hline CSS071218:124027-150558 & CSS1240-15 & $12: 40: 27$ & $-15: 05: 58$ & 2012 Mar 17 & SOAR & 3600 & $\mathrm{E}$ \\
\hline MLS110329:125118-020208 & MLS1251-02 & $12: 51: 18$ & $-02: 02: 08$ & 2012 Apr 23 & SOAR & 8100 & $\mathrm{E}$ \\
\hline SSS110724:135915-391452 & SSS1359-39 & $13: 59: 15$ & $-39: 14: 52$ & 2012 Apr 22 & SOAR & 1440 & $\mathrm{D} / \mathrm{IP}$ \\
\hline MLS110301:140203-090329 & MLS1402-09 & $14: 02: 03$ & $-09: 03: 29$ & 2012 Apr 23 & SOAR & 8100 & $\mathrm{E}$ \\
\hline MLS100617:140447-152226 & MLS1404-15 & $14: 04: 47$ & $-15: 22: 26$ & 2012 Mar 17 & SOAR & 3600 & $\mathrm{E}$ \\
\hline SSS100507:144833-401052 & SSS1448-40 & $14: 48: 33$ & $-40: 10: 52$ & 2012 Mar 26 & OPD & 1800 & PRG \\
\hline CSS100216:150354-220711 & CSS1503-22 & $15: 03: 54$ & $-22: 07: 11$ & 2012 Mar 16 & SOAR & 3600 & $\mathrm{P}$ \\
\hline MLS110526:151937-130602 & MLS1519-13 & $15: 19: 37$ & $-13: 06: 02$ & 2012 Mar 18 & SOAR & 3600 & RRL \\
\hline MLS110609:160907-104013 & MLS1609-10 & $16: 09: 07$ & $-10: 40: 13$ & 2012 Apr 22 & SOAR & 3600 & $\mathrm{P}$ \\
\hline CSS080606:162322+121334 & $\mathrm{CSS} 1623+12$ & $16: 23: 22$ & $+12: 13: 34$ & 2012 Apr 22 & SOAR & 8100 & $\mathrm{D}$ \\
\hline SSS100804:163911-235804 & SSS1639-23 & $16: 39: 11$ & $-23: 58: 04$ & 2012 Sep 03-04 & OPD & 2700 & PRG \\
\hline CSS110623:173517+154708 & $\mathrm{CSS} 1735+15$ & $17: 35: 17$ & $+15: 47: 08$ & 2012 Apr 23 & SOAR & 1440 & $\mathrm{D}$ \\
\hline 1RXS J174320.1-042953 & 1RXS1743-04 & $17: 43: 20$ & $-04: 29: 57$ & 2012 Mar 16 & SOAR & 720 & $\mathrm{P}$ \\
\hline 1RXS J192926.6+202038 & $1 \mathrm{RXS} 1929+20$ & $19: 29: 28$ & $+20: 20: 35$ & 2012 Apr 23 & SOAR & 1440 & $\mathrm{D}$ \\
\hline SSS110625:194030-633056 & SSS1940-63 & $19: 40: 30$ & $-63: 30: 56$ & 2012 Sep 03 & OPD & 2700 & PRG \\
\hline SSS100805:194428-420209 & SSS1944-42 & $19: 44: 28$ & $-42: 02: 09$ & 2012 Apr 22 & SOAR & 3600 & $\mathrm{P}$ \\
\hline SSS110526:195648-603430 & SSS1956-60 & $19: 56: 48$ & $-60: 34: 30$ & 2012 Apr 21 & SOAR & 720 & $\mathrm{P}$ \\
\hline USNO-A2.0 0825-18396733 & USNO0825-18 & $20: 31: 38$ & $-00: 05: 11$ & 2012 May 20-Jun 25 & SOAR & 3600 & $\mathrm{P}$ \\
\hline SSS110526:204247-604523 & SSS2042-60 & $20: 42: 47$ & $-60: 45: 23$ & 2012 Aug 10 & SOAR & 3600 & $\mathrm{HA}$ \\
\hline MLS100706:204358-194257 & MLS2043-19 & $20: 43: 58$ & $-19: 42: 57$ & 2012 Aug 10 & SOAR & 540 & $\mathrm{D} / \mathrm{IP}$ \\
\hline MLS111021:204455-162230 & MLS2044-16 & $20: 44: 55$ & $-16: 22: 30$ & 2012 Sep 03 & OPD & 2700 & RRL \\
\hline MLS101102:205408-194027 & MLS2054-19 & $20: 54: 08$ & $-19: 40: 27$ & 2012 Aug 10 & SOAR & 1800 & $\mathrm{D} / \mathrm{IP}$ \\
\hline CTCV J2056-3014 & CTCV2056-30 & $20: 56: 52$ & $-30: 14: 38$ & 2012 Sep 04 & OPD & 2700 & $\mathrm{D} / \mathrm{IP}$ \\
\hline CSS110513:210846-035031 & CSS2108-03 & $21: 08: 46$ & $-03: 50: 31$ & 2012 May 20-Jun 25 & SOAR & 3600 & $\mathrm{D}$ \\
\hline MLS100620:213227-150523 & MLS2132-15 & $21: 32: 27$ & $-15: 05: 23$ & 2012 Aug 10 & SOAR & 900 & RRL \\
\hline CSS100624:220031+033431 & $\mathrm{CSS} 2200+03$ & $22: 00: 31$ & $+03: 34: 31$ & 2012 Aug 14 & SOAR & 3600 & $\mathrm{D} / \mathrm{IP}$ \\
\hline 1RXS J222335.6+074515 & $1 \mathrm{RXS} 2223+07$ & $22: 23: 34$ & $+07: 45: 19$ & 2012 Aug 25 & SOAR & 8100 & $\mathrm{D}$ \\
\hline MLS100906:223034-042347 & MLS2230-04 & $22: 30: 34$ & $-04: 23: 47$ & 2012 Aug 25 & SOAR & 1440 & $\mathrm{E}$ \\
\hline SSS110720:224200-662512 & SSS2242-66 & $22: 42: 00$ & $-66: 25: 12$ & 2012 Sep 03-04 & OPD & 2700 & $\mathrm{D} / \mathrm{IP}$ \\
\hline CSS111021:231909+331540 & CSS2319+33 & $23: 19: 09$ & $+33: 15: 40$ & 2012 Sep 04 & OPD & 2700 & $\mathrm{D} / \mathrm{IP}$ \\
\hline
\end{tabular}

${ }^{\text {a }}$ P: polar candidate, D: disk system, D/IP: IP candidate, HA: system with high-state accretion disk, N: Nova, RRL: RR Lyrae star, PRG: Mira or low-amplitude Pulsating Red Giants star, E: extragalactic source. 
non-sinusoidal signals, as in the case of eclipsing binaries or pulsating stars.

An assessment of the FAP of detecting a signal due to statistical fluctuations of the power spectrum follows Scargle (1982, section III.c). The level of Lomb power $z_{0}$ correspondent to a false alarm probability $p_{0}$ is

$$
z_{0}=-\log \left[1-\left(1-p_{0}\right)^{1 / N_{f}}\right],
$$

where $N_{f}$ is the number of independent frequencies in the Lomb spectrum. For unequally spaced data, $N_{f}$ is not well defined, but overestimating it increases $z_{0}$, that is, makes the correspondent power level higher. We surely are overestimating this value, since with $N_{f}=1 \times 10^{6}$, the Lomb spectrum is finely sampled.

The uncertainty in period for the detected peaks in the Lomb spectrum, $\sigma_{P}$, follows the derivation of Kovacs (1981) and is very similar to an expression in Horne \& Baliunas (1986),

$$
\sigma_{P}=\frac{1}{2 \pi} \sqrt{\frac{24}{N}} \frac{P^{2}}{T} \frac{\sigma}{R}
$$

where $R$ is the semi-amplitude of the signal, $\sigma^{2}$ is the variance of the noise around the signal, $T$ is the span of the light curve and $N$ is the number of points in it.

The relevant results are presented for each object in Section 6.

\section{OBSERVATIONAL CHARACTERIS- TICS OF MAGNETIC CVS}

In this section, we describe the criteria used to classify an object as a $\mathrm{mCV}$ candidate. They are broadly consistent with previous studies in the field (e.g., Szkody et al. 2002, 2003, 2004, 2005, 2006, 2007, 2009, 2011, 2014; Thorstensen \& Skinner 2012; Thorstensen et al. 2016; Breedt et al. 2014; Woudt et al. 2012; Coppejans et al. 2014).

Our spectra, shown in Figure 1, allow us to differentiate CVs (Sects. 6.1 to 6.4) from stellar objects without accretion because of the lack of emission lines in the latter. Moreover, active galactic nuclei sources (Sec. 6.5) are also easily differentiated from CVs because of the redshifted, broad permitted lines. On the other hand, the distinction between magnetic and non-magnetic $\mathrm{CVs}$ is much more challenging. Hence this section concentrates on the observed characteristics of mCVs.

Usually, the confirmation of magnetic accretion, and therefore the classification as a $\mathrm{mCV}$, cannot be achieved with a single observational technique. The two kinds of observations that definitively confirm the presence of a magnetic white dwarf are the detection of polarized emission or Zeeman-splitting of spectral lines. But these observations are very time-consuming, particularly for IP systems in which these signals are barely discernible due to the masking caused by the contribution of the disk emission. Hence, to maximize the efficiency of finding $\mathrm{mCVs}$ in this work, we performed exploratory spectroscopy of selected objects. The spectral features and other properties of the objects are then analyzed in order to verify if they remain as $\mathrm{mCV}$ candidates. The characteristics used to this classification are discussed below. In many cases, a final classification as $\mathrm{mCV}$ can only be achieved with complementary observations, which are beyond the scope of this paper.

The mCV spectra typically do not show conspicuous contribution from the stellar components of the system: the continuum is usually flat or blue. When in low state, the optical spectrum of polars may show cyclotron humps (e.g., Thomas et al. 2012), which are not observed in IPs.

The most conspicuous spectral signature of accretion is the presence of emission lines, mainly of hydrogen Balmer series. However, this is a necessary but not sufficient criterion for mCVs classification. Magnetic CVs usually present highionization lines of He II and the C III/N III blend at $4650 \AA$ originated in the accretion column, although these features may be present in novalike and dwarf nova systems as well (Warner 1995). Specifically, the equivalent width of the hydrogen Balmer and He II $4686 \AA$ lines were proposed as criteria for the classification of magnetic CVs. According to Silber (1992) - as cited by Mukai's Intermediate Polars Homepage ${ }^{5}$ - IPs are characterized by $\mathrm{H} \beta$ equivalent width larger than $20 \AA$ and He II $4686 \AA / \mathrm{H} \beta$ larger than 0.4 . However, as it will be shown, these quantities should be under-

\footnotetext{
${ }^{5}$ http://asd.gsfc.nasa.gov/Koji.Mukai/iphome/iphome. html
} 
stood as suggestive of magnetic accretion, but not a sine qua non condition.

The line profiles are also useful to the object classification. Polars present narrow spectral lines, due to the absence of an accretion disk. Although low inclination non-magnetic CVs can also display narrow emission lines, polar lines are generally asymmetric, because they are composed of distinct components originated from different parts of the accretion flow and from the illuminated secondary face. IPs, however, can present line profiles very similar to those of non-magnetic CVs, with large widths and double peaks.

We have searched for high-energy counterparts because magnetic CVs are frequently detected in $\mathrm{X}$-ray surveys. In fact, a large number of magnetic CVs have been discovered by X-ray all-sky surveys, as ROSAT (e.g., Beuermann \& Thomas 1993). Magnetic CVs have higher X-ray to optical flux ratios than dwarf novae in quiescence (e.g., Beuermann \& Thomas 1993; Fertig et al. 2011; Szkody et al. 2011). This information and the hardness ratios, for objects having ROSAT counterparts, were used to provide a hint on whether an object might be a mCV (e.g., Hümmerich et al. 2014). However, some confirmed mCVs are not bright enough to be detected by the ROSAT survey (e.g., Silva et al. 2015; Margon et al. 2014). Figure 2 of Beuermann \& Thomas (1993) shows that polars with the lowest X-ray to optical flux ratio can be missed by ROSAT if they have $\mathrm{V}>$ $19 \mathrm{mag}$. The limit for IPs is even more restrictive: $\mathrm{V}>17 \mathrm{mag}$.

From a photometric point of view, polars alternate high and low brightness states in optical and X-rays with drops of $1-3$ magnitudes, probably associated to variations in the mass-transfer rate. They do not present outbursts, unlike some IPs. In short time-scales, mCV's fluxes vary periodically with the WD spin. The modulation of polars can have an amplitude as high as 1-2 magnitudes and their periods are around hours because the WD spin is synchronized with the orbital revolution. IPs have smaller amplitudes (tenths of magnitudes) and smaller periods (minutes). We searched for periodic variability in all stellar objects, as described in Sec. 4. However, the shape of the light curves vary from cycle to cycle due to flickering, which can hamper the discovery of periodic variability in long-term and sparse pho- tometric data, as CRTS.

The summary of the above discussion is that polars are more easily identified in our sample than IPs, because polars lack an accretion disk in such way that the accretion column dominates their emission. In contrast, IPs have a large contribution from the disk, which makes them observationally similar to non-magnetic CVs. Moreover, the X-ray emission and high-ionization emission lines are not exclusive properties of $\mathrm{mCVs}$ while, on the other hand, some $\mathrm{mCV}$ s are not detected in X-rays (e.g., Silva et al. 2015; Margon et al. 2014). Therefore, these observables were considered in the proposed classification of each object, but they are not definite criteria.

\section{CLASSIFICATION RESULTS}

\subsection{Polar Candidates}

This section comprises the polar candidates, or diskless accretors. The other mCVs - IP candidates - are placed in the next section together with other disk-accretion systems. Table 2 presents the FWHM and the equivalent width of the Balmer and He II $4686 \AA$ lines of the objects classified as CVs in this work.

\subsection{1. $M L S 0227+13(M L S 110213: 022733+130617)$}

Its CRTS light curve ranges from 16.5 to 19.5 mag on timescales of days. The spectrum is typical of polars with $\mathrm{H} \beta$ more intense than $\mathrm{H} \alpha$. The He II $4686 \AA$ emission line is as intense as $\mathrm{H} \beta$ (Table 2) and the high-ionization Bowen C III/N III is also present. In our follow-up observational study, Silva et al. (2015) confirmed MLS0227+13 as an eclipsing polar with an orbital period of $3.787 \mathrm{~h}$, using polarimetric, photometric and spectroscopic data. The system has no X-ray counterpart, which can be explained by its large distance from us (Silva et al. 2015).

\subsection{2. $C S S 0357+10(C S S 091109: 035759+102943)$}

CSS0357+10 was detected as a transient by CRTS on 2009 November 9. The V magnitudes are highly variable, spanning from 16.5 to $21 \mathrm{mag}$ in short timescales. Schwope \& Thinius (2012) performed a time-resolved photometric study of this target and found a periodicity of $114 \mathrm{~min}$ with 0.8 mag amplitude, interpreted as the orbital pe- 

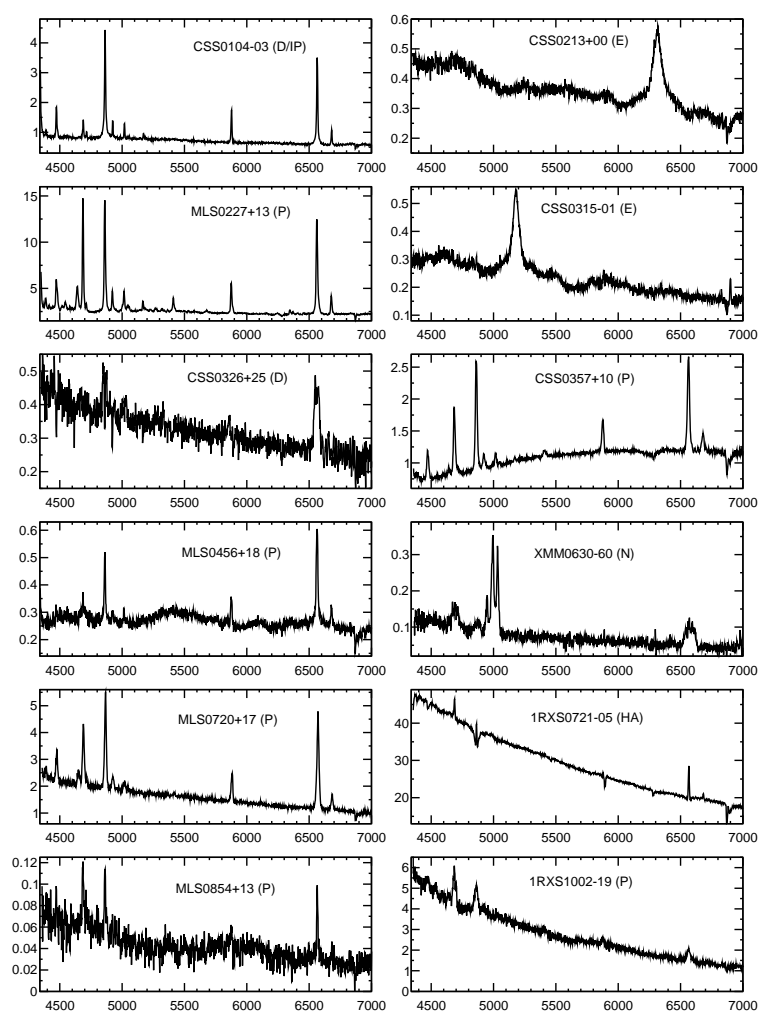

Fig. 1. - Identification spectra arranged by right ascension. The vertical axes are $\mathrm{F}_{\lambda}$ in units of $10^{-16}$ erg $\mathrm{cm}^{-2} \mathrm{~s}^{-1} \AA^{-1}$ and the horizontal axes are Wavelengths, in $\AA$. 


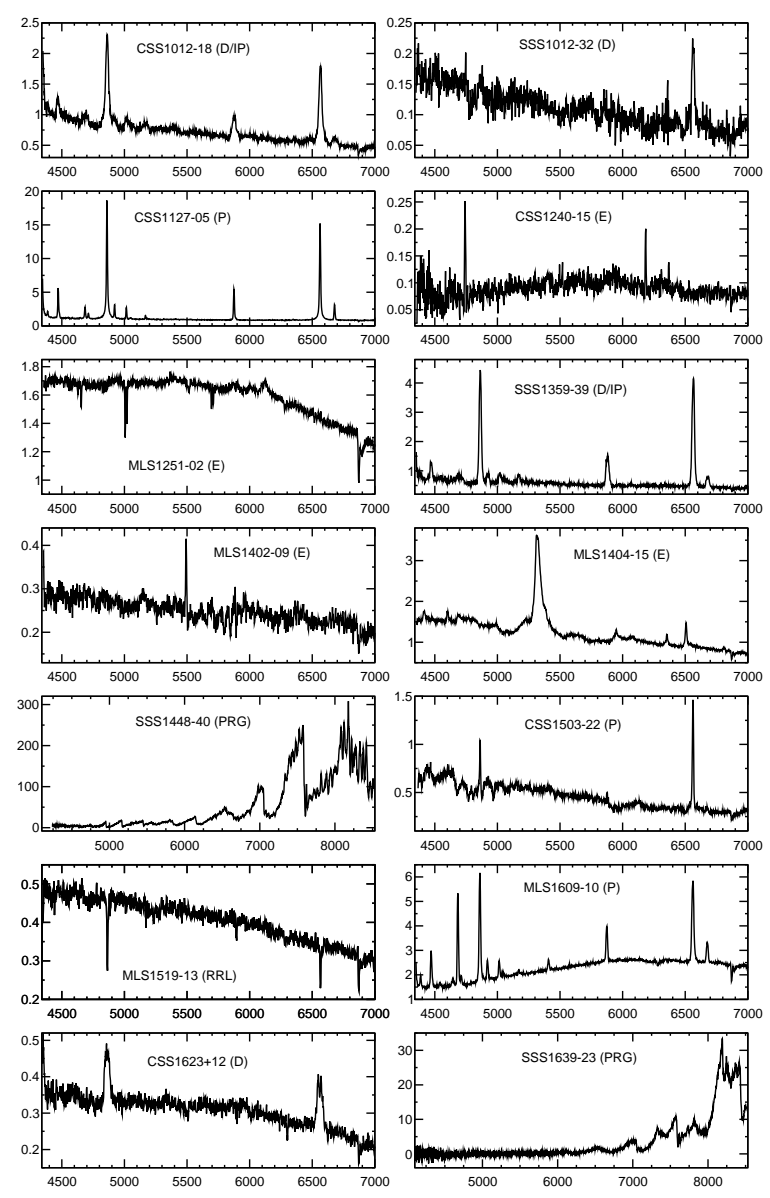

Fig. 1.-Continued 

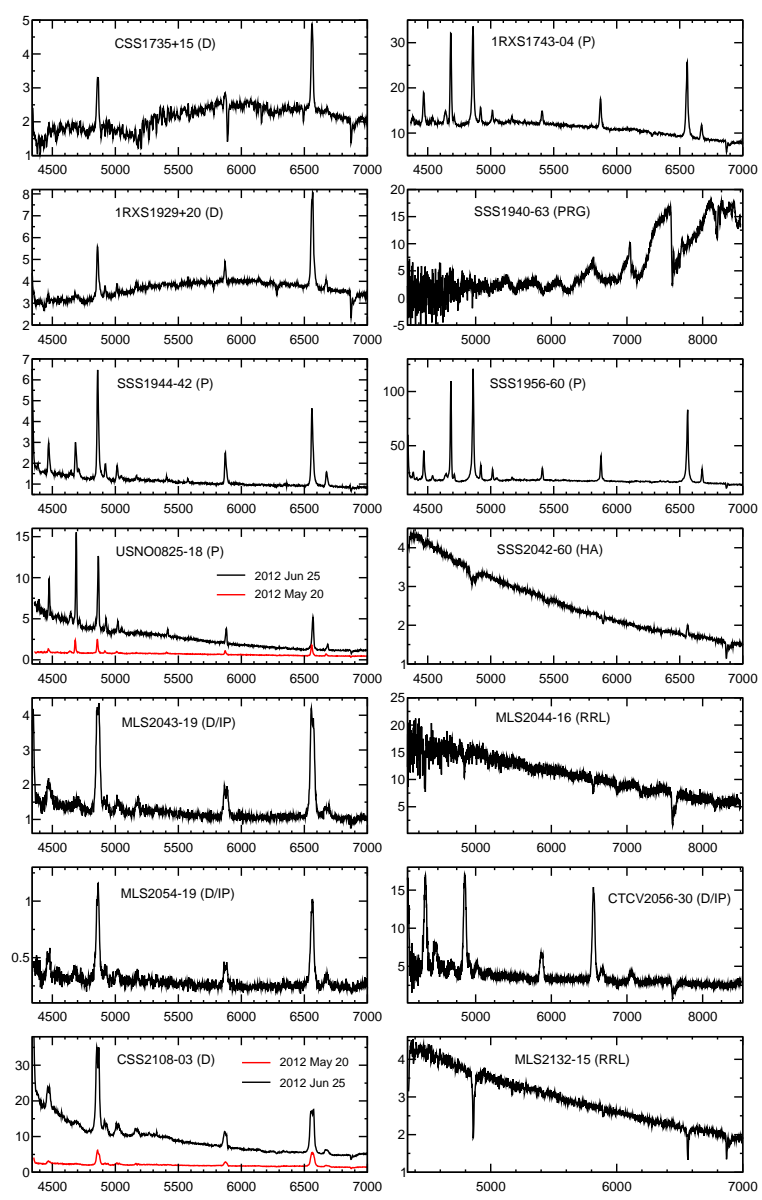

Fig. 1.-Continued
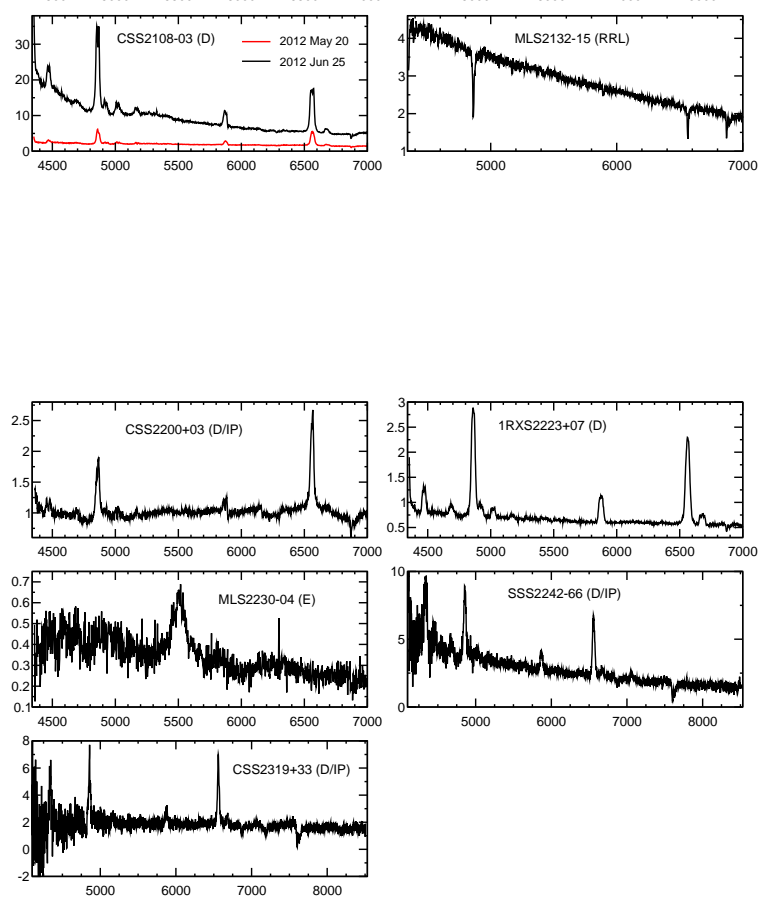

$5000 \quad 6000$

Fig. 1.-Continued 
riod of a non-eclipsing polar candidate. Recently, Thorstensen et al. (2016) obtained spectroscopic time series of this source and corroborated the 114 min orbital period. This object is also detected in X-rays (ROSAT, XMM-Newton), presenting large variability (Schwope \& Thinius 2012).

The identification spectrum is dominated by intense Balmer and He II $4686 \AA$ lines. It also presents He I and weak He II $5411 \AA$, Bowen C III/N III complex and Fe II $5170 \AA$ emissions. The Balmer and He II $4686 \AA$ lines are asymmetric, with extended red wings, and $\mathrm{H} \beta$ is more intense than $\mathrm{H} \alpha$ (Table 2), confirming the polar classification.

\subsection{3. $M L S 0456+18(M L S 101203: 045625+182634)$}

This object was detected as a variable star on 2013 December 10 by the CRTS. Its light curve spans from 18.5 to $21.5 \mathrm{mag}$, reaching $1 \mathrm{mag}$ variation amplitude in timescales of hours. Narrow Balmer emission lines dominate the identification spectrum, which also presents He I and He II 4686 $\AA$ lines. The latter line consists of a broad component $(\mathrm{EW}=18 \AA)$ superimposed on a very narrow central emission $(\mathrm{EW}=1 \AA)$. The composite line profile, the $\mathrm{EW}$ of $\mathrm{H} \beta$, and the ratio between He II $4686 \AA$ and $\mathrm{H} \beta$ (Table 2) suggest a mCV classification. The continuum shows humps that may be associated to cyclotron harmonics. This property and the absence of an X-ray counterpart are consistent with a low accretion-rate polar or a pre-polar system (e.g., Schmidt et al. 2005). The power spectrum of the CRTS data has its highest peak at $0.09851004 \mathrm{~d}(2.36 \mathrm{~h})$, which has a FAP smaller than $0.0001(=0.01 \%)$. The formal error of this period is $1.3 \times 10^{-7} \mathrm{~d}$ (see Section 4). The corresponding phase diagram (Figure 2) is compatible with the light curve of a polar system. This period places MLS0456+18 inside the period gap of CVs. As several objects in this work, a definite classification demands more observations.

\subsection{4. $M L S 0720+17(M L S 101226: 072033+172437)$}

The CRTS light curve of MLS0720+17 presents 0.5 mag amplitude variability around $18.5 \mathrm{mag}$, occasionally dropping to 20-21 mag, possible during eclipse events (Drake et al. 2014). The Goodman identification spectrum is typical of polars, with Balmer, He II $4686 \AA$, C III/N III and He I narrow emission lines superimposed on a continuum with a blue slope. The He I line profiles are asymmetric with extended blue wings in this spectrum. No X-ray source was found near its coordinates. The time series analysis applied to the CRTS data does not show any periodic signal that could explain the excursions to lower fluxes. Hardy et al. (2016) have recently presented a light curve spanning approximately $1 \mathrm{~h}$. It shows a modulation that the authors interpret as an eclipse. We consider it can arise from cyclotron emission, as usually seen in polars. Moreover, the narrow spectral lines are inconsistent with an eclipsing system having a disk.

\subsection{5. $M L S 0854+13(M L S 12012 \%: 085402+133633)$}

This target matches a blue stellar counterpart in the SDSS, with $u=20.18, g=20.58, r=20.43$, $i=20.28$, and $z=20.06$ in DR8. It presents two brightness levels with averages 19.7 and $21.5 \mathrm{mag}$ in the CRTS data. It is associated with the X-ray source 1WGA J0854.0+1336 in the WGA Catalog of ROSAT Point Sources ${ }^{6}$. This object is also classified as BOSS J085402.10+133632.8, a $\mathrm{z}=2$ QSO in the MILLIQUAS Catalog V4.7 ${ }^{7}$, with no SDSS spectrum available. However, our spectrum is completely consistent with a Galactic object, being similar to the spectrum of a polar in low accretion state, with cyclotron humps and emission lines of He II $4686 \AA, \mathrm{H} \beta$ and $\mathrm{H} \alpha$ on a blue continuum. No periodic signal was found in the CRTS time series analysis.

\subsubsection{RXS1002-19 (1RXS J100211.4-192534)}

This source was discovered by ROSAT (Beuermann \& Thomas 1993) and classified as a polar with an X-ray orbital period of 106 min (Beuermann \& Schwope 1994). 1RXS1002-19 was observed with XMMNewton (Ramsay \& Cropper 2003) and the resulting X-ray light curve suggests that the source of radiation is located in one hemisphere of the WD, while modeling of the X-ray spectrum yields a WD mass close to $0.5 \mathrm{M}_{\odot}$.

Our optical spectrum of 1RXS1002-19, the first for this source, has a strong blue continuum. The He II $4686 \AA$ line is more intense than $\mathrm{H} \beta$, which in

\footnotetext{
${ }^{6}$ http://heasarc.gsfc.nasa.gov/wgacat/

${ }^{7}$ http://heasarc.gsfc.nasa.gov/w3browse/all/ milliquas. html
} 
turn is more intense than $\mathrm{H} \alpha$ (Table 2). He I features are also present in emission. The FWHM of the $\mathrm{H} \beta$ and He II $4686 \AA$ lines are around $2000 \mathrm{~km}$ $\mathrm{s}^{-1}$ (Table 2), which is unusual for a polar. The equivalent width of the $\mathrm{H} \beta$ emission is $11 \AA$, smaller than the value of $20 \AA$ from the Silber's criterion (Silber 1992) for a magnetic CV classification. Our spectrum converts to $\mathrm{V}=18 \mathrm{mag}$. This locates the object in the polar systems region of X-ray count rate versus optical mag diagram, according to Figure 2 of Beuermann \& Thomas (1993).

\subsubsection{CSS1127-05 (CSS110225:112749-054234 = SSS120624:112749-054236)}

CSS1127-05 has a blue point source counterpart in the SDSS, with $u=20.00, g=20.12, r=$ $20.09, i=20.28$ and $z=20.15$ in DR8. The CRTS light curve clearly shows two distinct brightness states with average magnitudes of 18.5 and 20.5. The exploratory spectrum has high signal to noise ratio, displaying strong and narrow Balmer lines in emission on a flat continuum, besides less intense He II $4686 \AA$ and He I. H $\beta$ is more intense than $\mathrm{H} \alpha$. The $\mathrm{H} \alpha \mathrm{EW}(204 \AA)$ is the largest among the objects we classify as CV (Table 2). In spite of the low ratio between He II $4686 \AA$ and $\mathrm{H} \beta$ (0.16), considering the Silber's criterion (Silber 1992), we suggest it is a polar due to the presence of high and low-brightness states. However, we do not discard a possible IP classification since these objects may also present low states, although less commonly. No significant periodicity was found in the CRTS data. The system has no X-ray counterpart.

\subsubsection{CSS1503-22 (CSS100216:150354-220711 = SSS100512:150354-220710)}

The light curve of CSS1503-22 presents two alternating brightness states at average magnitudes 19.5 and 17.5 , both states with dispersion of 1 to 1.5 mag. Woudt et al. (2012) performed photometric monitoring of CSS1503-22 in March and April 2010 on five runs summing up $15 \mathrm{~h}$ of observations when it was at high state. They found a period of $133.38 \mathrm{~min}$ and, from its photometric behavior and the detection as a Swift X-ray source, they suggested it to be a polar. The flux of our SOAR spectrum corresponds to $19.7 \mathrm{mag}$, which is consistent with the CRTS low state. The spectrum presents absorption features from 4500 to
$6000 \AA$, which we interpret as from the stellar components. Some absorptions occur at typical wavelengths of $\mathrm{TiO}$ bands from a M2 - M5 secondary star (see, for instance, Mennickent et al. 2002 and Gray \& Corbally 2009). The absorptions near $\mathrm{H} \beta$ and to the blue of $\mathrm{H} \alpha$ are probably due to Zeeman splitting in the photosphere of the white dwarf. See, for instance, the SDSS J214930.74072812.0 spectrum and the superimposed model atmosphere with $\mathrm{B}=44.7 \mathrm{MG}$ presented in Külebi et al. (2009). The spectrum also displays narrow Balmer lines, possibly with blue wings, and $\mathrm{H} \beta$ being half the intensity of $\mathrm{H} \alpha$ (Table 2). The He I $5876 \AA$ line is also present. The Zeeman absorptions hamper the detection of possible He II $4686 \AA$ or Bowen complex emissions. The EW of $\mathrm{H} \beta$ is $16 \AA$, slightly smaller than the value of $20 \AA$ from the Silber's criteria (Silber 1992), but the accuracy of the measured EW can be affected by the cited absorptions.

\subsubsection{MLS1609-10 (MLS110609:160907-104013)}

The CRTS light curve of this object has measurements scattered from 16.8 to $20.5 \mathrm{mag}$. The exploratory spectrum presents intense and narrow Balmer lines in emission. These lines are slightly asymmetrical, presenting extended red wings. $\mathrm{H} \beta$ is more intense than $\mathrm{H} \alpha$, while He II $4686 \AA$ is almost as intense as $\mathrm{H} \beta$ (Table 2). The Bowen complex at $4630-4650 \AA$ is clearly visible. The spectrum shown in Figure 1 is the average of three consecutive spectra, obtained with 20 min intervals. They show a remarkable variability in the continuum, hence they are individually presented in Figure 3. We performed a $3.6 \mathrm{~h}$ span photometric time-series of this source on 2014 April 28, using the $1.6 \mathrm{~m} \mathrm{P \& E}$ telescope at OPD and the Andor iKon-L936BV camera, obtaining 39 images with $240 \mathrm{~s}$ exposure time through the V filter. The differential photometry was converted to $\mathrm{V}$ magnitude using as reference the star NOMAD1 07930295201, with $m_{v}=16.51$ (Zacharias et al. 2004).

The OPD lightcurve is consistent with a polar classification and shows a modulation with a period around $0.077 \mathrm{~d}$ and $0.60 \pm 0.05 \mathrm{mag}$ amplitude. In the search for periodic signals in the CRTS data, the PDM method yielded a period of $\mathrm{P}=0.075439 \pm 0.000001 \mathrm{~d}$, compatible with the OPD period. Figure 4 shows both OPD and CRTS light curves folded with $\mathrm{P}=0.075439 \mathrm{~d}$ and 
epoch $\mathrm{T}_{\min }=2456776.7108$ HJD. The average magnitude of the OPD data is $\mathrm{V}=21.3$, therefore the source was caught in a very low state, whereas the individual spectra correspond to 17.8 and 19 $\mathrm{V}$ mag. The system has no X-ray counterpart.

\subsubsection{RXS1743-04 (1RXS J174320.1-042953 $=$ USNO-B1.0 0855-0326594)}

This CV was discovered by Denisenko \& Sokolovsky (2011) among X-ray sources in the ROSAT catalog. Denisenko \& Martinelli (2012) performed time-resolved photometry and suggested the target to be a polar or IP, based on the amplitude (0.8 mag in white light) and shape of the light curve. They determined an orbital period of 2.08 h.

Our exploratory spectrum is typical of polars, with very intense He II $4686 \AA$ and $\mathrm{H} \beta$ more intense than $\mathrm{H} \alpha$. The $\mathrm{C}$ III/N III complex is also present and the continuum increases to the blue. Our spectrum corresponds to $\mathrm{V}=16.3 \mathrm{mag}$.

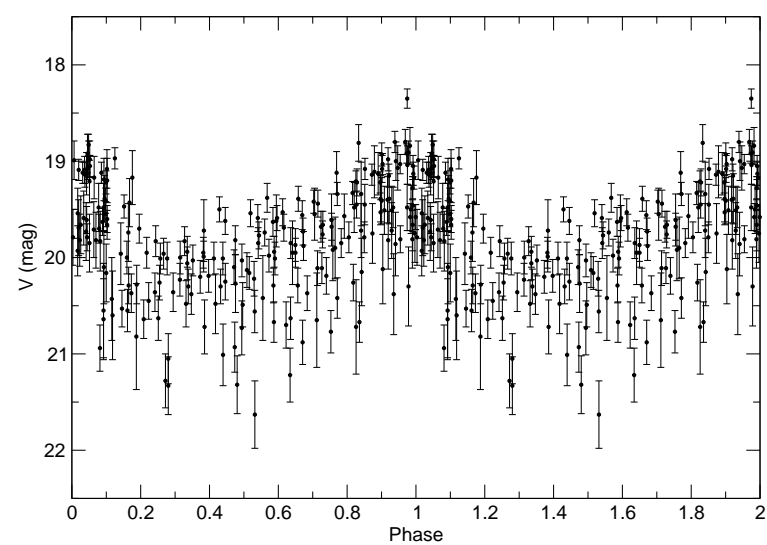

Fig. 2.- CRTS light curve of MLS0456+18 folded with the period of $0.09851004 \mathrm{~d}$.

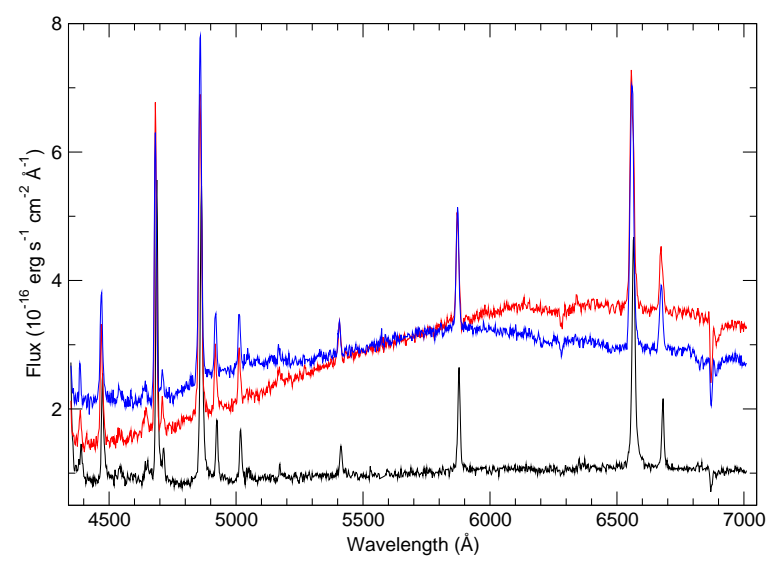

Fig. 3.- Three consecutive (black, red, blue) spectra of MLS1690-10 obtained with 20 min intervals. 


\subsubsection{SSS1944-42 (SSS100805:194428-420209)}

The CRTS light curve of this object presents two brightness levels separated by 2 mag and short timescale variability. Coppejans et al. (2014) performed photometric monitoring during the bright state and classified it as a polar from its light curve, which is modulated with a period of $1.53 \mathrm{~h}$. Our spectrum was obtained in the low-brightness state $(\mathrm{V} \approx 19 \mathrm{mag})$ and presents narrow, single peaked Balmer, He I and He II $4686 \AA$ emission features over a blue continuum. The Balmer and He II lines have extended red wings and $\mathrm{H} \beta$ is $35 \%$ more intense than $\mathrm{H} \alpha$ (Table 2). The $\mathrm{C}$ III/N III Bowen complex at $4640 \AA$ and the lines of Fe II $5169 \AA$ and He II $5412 \AA$ are also visible. The system has no X-ray counterpart.

\subsubsection{SSS1956-60 (SSS110526:195648-603430)}

This source presents many kinds of variability in the CRTS light curve: long (years) and short (one month) term variations between magnitudes 18 and 15 , and $1 \mathrm{mag}$ variations in timescales of a few days. SSS1956-60 is classified as the novalike system CV V348 Pav (Downes et al. 2001) and is also identified as V1956-6034, a polar candidate by its spectrum (Drissen et al. 1994). Our exploratory spectrum displays narrow Balmer, He I and He II emission lines. The line of He II $4686 \AA$ is almost as intense as $\mathrm{H} \beta$, which in turn is more intense than $\mathrm{H} \alpha$ (Table 2). Also present in the spectrum are the lines of the Bowen complex, He II 4541 and $5412 \AA$ and Fe II $5169 \AA$. In the spectrum obtained in 1992 by Drissen et al. (1994), the ratio of the intensities $\mathrm{H} \beta / \mathrm{He}$ II $4686 \AA$ is 0.25 , much smaller than the value of 0.91 we measured in our spectrum from 2012. An X-ray counterpart was detected with 1.8 counts $\mathrm{sec}^{-1}$ total band count rate at these coordinates by XMM-Newton in the slew data mode, and was registered as XMMSL1 J195647.7-603425 in the XMM-Newton Slew Survey Clean Source Catalog (Saxton et al. 2008).

\subsubsection{USNO0825-18 (USNO-A2.0 0825-18396733)}

The discovery of this polar candidate is reported by Kryachko et al. (2010). From the R light curve, they obtained an orbital period of $0.0848 \mathrm{~d}(2.035 \mathrm{~h})$, and their spectrum shows He II $4686 \AA$ almost as intense as $\mathrm{H} \beta$ and also He I and the Bowen blend. Afanasiev et al. (2015) performed polarimetric observations of this source and found variable circular polarization reaching $-31 \%$.

We obtained exploratory spectra of USNO082518 in two occasions in 2012,35 days apart. In the second run, the flux in the blue was 6 times more intense than in the first observation (Figure 1) and the He II $4686 \AA$ to $\mathrm{H} \beta$ intensity ratio increased from 0.97 to 1.23 (Table 2).

\subsection{Systems with Disks}

In this section, we present the systems with accretion disks. Possible IPs are also included here.

\subsubsection{CSS0104-03 (CSS091009:010412-031341)}

This object was discovered and classified as an eclipsing CV by CRTS on 2009 October 9 in a single outburst when it reached $\mathrm{V}=17.2 \mathrm{mag}$. CRTS data span from 2005 to 2015, during which the object showed average $\mathrm{V}=19 \mathrm{mag}$ and $\sim 1$ mag variability in timescales of hours, with occasional 21 mag excursions. Another outburst was recorded by ASAS at 16.39 mag on 2013 August 27 (Ohshima 2013). Szkody et al. (2014) present a spectrum with strong emission Balmer lines obtained on 2013 September 3 and classify the object as a dwarf nova, possibly eclipsing due to the dispersion of the CRTS quiescent light curve. This spectrum was taken in a state brighter than the quiescent flux in the CRTS light curve. The SDSS DR9 counterpart of CSS0104-03 presents $u=19.2, g=19.5, r=19.1, i=18.8$ and $z=18.9$.

Our Goodman spectrum shows narrow and single peaked Balmer, He I and He II $4686 \AA$ emission lines, $\mathrm{H} \beta$ being 1.26 times more intense than $\mathrm{H} \alpha$ (see Table 2). The continuum is flat with a slight slope to the blue. The Balmer lines have extended wings and are slightly asymmetrical. The average continuum flux in our spectrum is a factor of 4 less intense than the continuum flux in the spectrum published by Szkody et al. (2014), in which the line of He II $4686 \AA$ is only marginally detected. CSS0104-03 has the highest He II $4686 \AA$ EW among our sample of disk-accretion systems. Our spectrum has a flux level around 19 mag in the $\mathrm{V}$ band, hence it is consistent with the mean level of the CRTS light curve.

Our time series analysis of the CRTS data does not reveal any period which could explain the low 
TABLE 2

Full Width at Half Maximum (FWHM ${ }^{\mathrm{a}}$ ) and Equivalent Width (EW) of He II $4686 \AA$, H $\beta$ AND H $\alpha$ EMISSION LINES OF THE OBJECTS CLASSIFIED AS POLARS AND DISK SYSTEMS.

\begin{tabular}{|c|c|c|c|c|c|c|c|}
\hline Object & $\begin{array}{c}\text { FWHM He II } \\
\left(\mathrm{km} \mathrm{s}^{-1}\right)\end{array}$ & $\begin{array}{c}-\mathrm{EW} \text { He II } \\
(\AA)\end{array}$ & $\begin{array}{l}\text { FWHM H } \beta \\
\left(\mathrm{km} \mathrm{s}^{-1}\right)\end{array}$ & $\begin{array}{c}-\mathrm{EW} \mathrm{H} \beta \\
(\AA)\end{array}$ & $\begin{array}{c}\text { FWHM H } \alpha \\
\left(\mathrm{km} \mathrm{s}^{-1}\right)\end{array}$ & $\begin{array}{c}-\mathrm{EW} \mathrm{H} \alpha \\
(\AA)\end{array}$ & $\mathrm{He} \mathrm{II} / \mathrm{H} \beta$ \\
\hline CSS0104-03 & 490 & 6 & 580 & 59 & 450 & 63 & 0.32 \\
\hline MLS0227+13 & 630 & 47 & 700 & 66 & 600 & 76 & 1.01 \\
\hline CSS0326+25 & $\cdots$ & $\cdots$ & $\mathrm{p}^{\mathrm{b}}$ & $\mathrm{p}$ & 2380: & 47: & $\ldots$ \\
\hline $\mathrm{CSS} 0357+10$ & 1040 & 22 & 1050 & 40 & 900 & 30 & 0.73 \\
\hline MLS0456+18 & 750 & 13 & 730 & 20 & 690 & 23 & 0.71 \\
\hline MLS0720+17 & 1040 & 19 & 940 & 32 & 850 & 65 & 0.79 \\
\hline 1RXS0721-05 & 530 & 1 & 420 & 1 & 400 & 4 & 0.83 \\
\hline MLS0854+13 & 930 & 15 & 810 & 24 & 490 & 30 & 1.06 \\
\hline 1RXS1002-19 & 1850 & 12 & 2090 & 11 & 1200 & 10 & 1.17 \\
\hline CSS1012-18 & $\mathrm{p}$ & $\mathrm{p}$ & 1710 & 64 & 1250 & 78 & $\ldots$ \\
\hline SSS1012-32 & $\cdots$ & $\cdots$ & $\mathrm{p}$ & $\mathrm{p}$ & 1120: & 53: & $\ldots$ \\
\hline CSS1127-05 & 540 & 14 & 550 & 183 & 450 & 204 & 0.16 \\
\hline SSS1359-39 & $\mathrm{p}$ & $\mathrm{p}$ & 1240 & 157 & 1030 & 197 & $\ldots$ \\
\hline CSS1503-22 & $\cdots$ & $\ldots$ & 490 & 16 & 490 & 44 & $\ldots$ \\
\hline MLS1609-10 & 730 & 29 & 870 & 40 & 840 & 26 & 0.87 \\
\hline CSS1623+12 & $\cdots$ & $\cdots$ & 2670 & 19 & 2370 & 28 & $\cdots$ \\
\hline CSS1735+15 & $\cdots$ & $\cdots$ & 1250 & 23 & 840 & 24 & $\ldots$ \\
\hline 1RXS1743-04 & 760 & 21 & 880 & 32 & 730 & 36 & 0.96 \\
\hline $1 \mathrm{RXS} 1929+20$ & $\mathrm{p}$ & $\mathrm{p}$ & 1000 & 14 & 1020 & 30 & $\ldots$ \\
\hline SSS1944-42 & 950 & 16 & 890 & 65 & 770 & 65 & 0.46 \\
\hline SSS1956-60 & 600 & 48 & 700 & 80 & 570 & 59 & 0.91 \\
\hline USNO0825-18 (May 20) & 630 & 22 & 660 & 22 & 630 & 42 & 0.97 \\
\hline USNO0825-18 (Jun 25) & 580 & 27 & 690 & 26 & 640 & 38 & 1.23 \\
\hline SSS $2042-60$ & $\ldots$ & $\ldots$ & 860 & 1 & 630 & 2 & $\ldots$ \\
\hline MLS2043-19 & $\mathrm{p}$ & $\mathrm{p}$ & 2070 & 87 & 1650 & 91 & $\ldots$ \\
\hline MLS2054-19 & $\mathrm{p}$ & $\mathrm{p}$ & 1700 & 84 & 1311 & 93 & $\ldots$ \\
\hline CTCV2056-30 & $\mathrm{p}$ & $\mathrm{p}$ & 2430 & 124 & 1850 & 160 & $\ldots$ \\
\hline CSS2108-03 (May 20) & $\mathrm{p}$ & $\mathrm{p}$ & 1720 & 51 & 1520 & 81 & $\cdots$ \\
\hline CSS2108-03 (Jun 25) & $\mathrm{p}$ & $\mathrm{p}$ & 1930 & 66 & 1690 & 92 & $\ldots$ \\
\hline CSS $2200+03$ & $\mathrm{p}$ & $\mathrm{p}$ & 2010 & 32 & 1320 & 43 & $\ldots$ \\
\hline 1RXS2223+07 & $\mathrm{p}$ & $\mathrm{p}$ & 2010 & 90 & 1720 & 128 & $\ldots$ \\
\hline SSS2242-66 (Sep 03) & $\mathrm{p}$ & $\mathrm{p}$ & 2200 & 71 & 1890 & 125 & $\ldots$ \\
\hline SSS2242-66 (Sep 04) & $\mathrm{p}$ & $\mathrm{p}$ & 2300 & 56 & 1610 & 62 & $\ldots$ \\
\hline CSS2319+33 & $\mathrm{p}:$ & p: & 1360 & 144 & 1130 & 85 & $\cdots$ \\
\hline
\end{tabular}

${ }^{a}$ The FWHM were measured from Voigt profile fiting.

${ }^{\mathrm{b}}$ The symbol $\mathrm{p}$ means that the line is present in the spectrum but its $\mathrm{S} / \mathrm{N}$ is low and no measurements were possible. 
flux excursions as eclipses. The small widths of the lines are also inconsistent with an eclipsing system. So the system could be a near face-on dwarf nova. However, this classification does not explain the low flux measurements, the two-component emission lines, and the He II $4686 \AA$ line behavior, since its intensity was expected to be higher at the spectrum from Szkody et al. (2014) taken at a brighter state. The object has no X-ray counterpart. If we suppose that its low optical brightness is due to its distance, it is possible that its X-ray luminosity is not high enough to allow the object detection in X-ray surveys. This seems to be true for MLS0227+13 (Silva et al. 2015). Therefore, a magnetic classification is a reasonable possibility taking into account the observations. Moreover, if the brightest points correspond to eruptions, it could be an IP system, possibly similar to GK Per or EX Hya. Spectroscopic time series would be useful to a definite classification of this system.

\subsubsection{CSS0326+25 (CSS090922:032603+252534)}

The light curve of this source, obtained by CRTS, has a large amplitude of variation, from 18.5 to 20 mag. It might be originated by flips between two brightness states. The average spectrum presents broad emission lines of $\mathrm{H} \alpha$ and $\mathrm{H} \beta$. $\mathrm{H} \alpha$ shows a profile with two peaks, while $\mathrm{H} \beta$ is quite peculiar, with a strong central absorption at the rest position of the line, in the center of the broad emission. Also marginally detectable are absorption lines of the Na I doublet at 5890 - 5896 $\AA$ and the diffuse interstellar band (DIB) at 6284 $\AA$. Our time series analysis does not reveal any remarkable signal. This object has no evidence of being a magnetic system.

\subsubsection{CSS1012-18 (CSS120324:10121\%-182411 = SSS110203:101217-182411)}

This CRTS source has been discovered by SSS on 2011 February 3, receiving the ID SSS110203:101217182411 and was later detected by CSS, on 2012 March 24, when it was assigned as CSS120324:101217182411. The CRTS light curves varies between 18-20 mag with sporadic outbursts reaching 16-17 mag. Our spectrum displays a blue continuum and broad He I and Balmer emission lines, $\mathrm{H} \beta$ being more intense than $\mathrm{H} \alpha$. He II $4686 \AA$ is present as well. The spectrum flux corresponds to approximately $19.3 \mathrm{mag}$ in the $\mathrm{V}$ band, consistent with the quiescent flux level. Coppejans et al. (2016) classify it as a dwarf nova with 3 detected outbursts. Its spectrum resembles the spectrum of the IP candidate CTCV2056-30 (see section 6.2.11) and short-period IPs like SDSS J2333 (Southworth et al. 2007; Szkody et al. 2005) and HT Cam (Kemp et al. 2002), so we suggest that it is a member of this class. The String-Length spectrum of the CRTS data shows some structure, but they do not translate into discernible features in the folded diagrams.

\subsubsection{SSS1012-32 (SSS110504:101240-325831)}

This object is associated with the X-ray source 1RXS J101239.6-325839. The CRTS light curve has short term (days) variability between 17.3 and $19.5 \mathrm{mag}$, but no periodicity was found. Moreover, the CRTS light curve does not present DNtype eruptions. The spectrum has low $\mathrm{S} / \mathrm{N}$, but $\mathrm{H} \alpha$ is clearly seen as a broad emission line while $\mathrm{H} \beta$ is weak. The continuum is quite blue. Despite not showing spectral features characteristic of magnetic systems, its ROSAT colors match those sources. The spectrum shows modulations that might be due to absorptions features of a red stellar component, but we could not associate their wavelengths to features usually found in $\mathrm{CV}$ secondaries. We do not classify this system as a mCV because of the lack of spectral evidence.

\subsubsection{SSS1359-39 (SSS110724:135915-391452)}

SSS1359-39 was discovered by CRTS and its light curve has a quiescent brightness level at 19 mag with a dispersion of $1 \mathrm{mag}$, and sporadic outbursts at magnitudes $14-15$. The periodicities search had negative results. This source is associated to the X-ray source 1RXS J135915.6391447. The exploratory spectrum shows features related to an accretion disk, with intense and broad Balmer emission lines (FWHM > $1000 \mathrm{~km}$ $\mathrm{s}^{-1}$ - Table 2) along with He I, Fe I, Fe II and He II $4686 \AA$, and is very similar to the spectrum of the IP candidate CTCV2056-30 (section 6.2.11), so we classify it as a possible short-period IP.

\subsubsection{CSS1623+12 (CSS080606:162322+121334)}

Photometric monitoring from CRTS yields a flat light curve at $17 \mathrm{mag}$ with rapid and short occasional decreases of 3 magnitudes. SDSS reg- 
isters this object as a blue point source with $u=21.85, g=21.88, r=21.42, i=21.38$ and $z=21.25$. Drake et al. (2014) obtained a spectrum of this object in high-accretion state, with broad Balmer absorptions on a blue continuum. Our SOAR spectrum was obtained in a faint state $(\mathrm{V} \approx 20.2 \mathrm{mag})$ and shows the Balmer lines in emission. These lines are broad with double peak profiles, indicating a possible high orbital inclination. The system may be eclipsing, which would explain the rapid and deep drops in the CRTS light curve. The time series analysis of the CRTS data does not reveal any clear periodicity, probably because most of the fainter points are upper limits and are not included in the photometric CRTS data. If the object is indeed eclipsing, our spectrum (exposure time of $2700 \mathrm{~s}$ ) would have been taken predominantly during the eclipse, which should be relatively long. This source could be alternatively interpreted as a VY Scl novalike variable after its spectral and photometric behavior.

\subsubsection{CSS1735+15 (CSS110623:173517+154708)}

The light curve obtained by CRTS is nearly flat around 17 and $17.5 \mathrm{mag}$ with one episodic measurement at $14.3 \mathrm{mag}$ and another at $18 \mathrm{mag}$. Thorstensen \& Skinner (2012) obtained one spectrum in September 2011 in which $\mathrm{H} \alpha$ is visible in emission together with a K-star feature at $5168 \AA$. Kato et al. (2013) found two photometric periods ( $0.05436 \mathrm{~d}$ and $0.05827 \mathrm{~d}$ ) but the interpretation of them is still not clear. The authors suggested they could be related to superhumps. Spectral observations by Drake et al. (2014) reveal CSS1735+15 in eruption with a steep blue continuum and absorption features of $\mathrm{H} \gamma$ and $\mathrm{H} \delta$. The SOAR spectrum has the same flux level as the spectrum from Thorstensen \& Skinner (2012) and displays typical features of a K star and $\mathrm{Na}$ I absorption together with emission lines of H I and He I. Our spectrum has not enough coverage to allow luminosity class determination, so we cannot discard that the secondary is an evolved object. After the submission of this manuscript, Thorstensen et al. (2016) presented time-series spectroscopy and determined an orbital period of $8.48 \mathrm{~h}$ and a distance of $1830 \pm 330$ pc from a K4 \pm 1 spectral type secondary star. This large period challenges the interpretation of the modulations as superhumps, which occurs for systems below the period gap. CSS1735+15 is unlikely an mCV.

\subsubsection{RXS1929+20 (1RXS J192926.6+202038 $=U S N O-B 1.01103-0421031)$}

This is another CV discovered by Denisenko \& Sokolovsky (2011) among X-ray sources in the ROSAT cata$\log$. It was classified as a possible dwarf nova by its X-ray hardness ratio. Our exploratory spectrum is reddened, with emission lines of H I, He I and marginal evidence of He II $4686 \AA$, besides two DIBs at 5780 and $6284 \AA$. The feature at 5577 $\AA$ is associated to bad subtraction of the telluric [O I] line. This CV has no evidence of being a magnetic system.

\subsubsection{MLS2043-19 (MLS100706:204358-194257)}

MLS2043-19 was discovered as a transient optical source by CRTS. Its light curve has a quiescent level at $\sim 19.5 \mathrm{mag}$ and four registered outbursts, reaching 15 mag. The CRTS data do not present any evidence of eclipse or periodicity. The SOAR spectrum, on the other hand, presents broad and double-peaked Balmer and He I emission lines, which suggest a high orbital inclination system, besides weak He II $4686 \AA$ or C III/N III emission features over a blue continuum. It also reminds us the spectrum of the IP candidate CTCV2056-30 presented in section 6.2.11, so we suggest it is a short-period IP candidate.

\subsubsection{MLS2054-19 (MLS101102:205408-194027 $=$ CSS090829:205408-194027)}

This object, discovered by CRTS, has a bimodal light curve with a low-brightness state at $19.5 \mathrm{mag}$ with 1 mag variation and several outbursts reaching 18-16 mag. Coppejans et al. (2014) found a photometric period of $0.09598 \mathrm{~d}$, suggesting it to be associated to superhumps of a SU UMa type $\mathrm{CV}$, and estimated an orbital period of $0.0917 \mathrm{~d}$. Our exploratory spectrum shows double-peaked $\mathrm{H} \beta$ emission more intense than $\mathrm{H} \alpha$, besides $\mathrm{He} \mathrm{I}$ and He II $4686 \AA$ (and possibly He II $4542 \AA$ ), being similar to the spectrum of the IP candidate CTCV2056-30, which suggests possible magnetic accretion. Interestingly, if the photometric period was the orbital period, the object would be in the period gap of non-magnetic CVs. 


\subsubsection{CTCV2056-30 (CTCV J2056-3014= 1RXS J205652.1-301433)}

This object was discovered by Augusteijn et al. (2010) during the Calán-Tololo Survey. Their low-resolution spectra present Balmer and He I emission lines over a blue continuum. The radial velocity curves provided a period of $1.76 \mathrm{~h}$ assumed as the orbital period, while photometric monitoring yields an additional modulation with $\mathrm{P}=15.4$ min, leading to a highly probable IP classification. Augusteijn et al. (2010) also report some likely dwarf nova outbursts registered by ASAS and the ROSAT X-ray detection as 1RXS J205652.1-301433. Our spectrum shows $\mathrm{H} \beta$ as intense as $\mathrm{H} \alpha$ and relatively intense $\mathrm{He} \mathrm{I}$ and weak He II $4686 \AA$ lines. The continuum is flatter and about 3 times less intense in the blue than the spectrum from Augusteijn et al. (2010). The spectrum is quite similar to that of short-period IPs like SDSS J2333 (Southworth et al. 2007; Szkody et al. 2005), HT Cam (Kemp et al. 2002) and DW Cnc (Patterson et al. 2004; Rodríguez-Gil et al. 2004), among others. These objects present spectra that are indistinguishable from quiescent dwarf novae spectra and, therefore, show that a single optical spectrum is not enough to confirm the IP nature of a source. The definite IP classification demands the detection of the white dwarf spin period not synchronized with the orbital period.

\subsubsection{CSS2108-03 (CSS110513:210846-035031)}

This system was discovered by CRTS in May 2011, displaying a light curve with a quiescent level at $18 \mathrm{mag}$ and outbursts reaching $15 \mathrm{mag}$. Coppejans et al. (2014) performed time-resolved photometry in October 2011 and found eclipses, deriving an orbital period of $0.15699 \mathrm{~d}$. A period of $0.1569268 \mathrm{~d}$ was determined independently by Drake et al. (2014) from the photometric data in the CRTS itself, in both quiescence and outburst.

We obtained two exploratory spectra of CSS210803, in 2012 May 20 and 2012 June 25. The data display characteristic features of a disk system. Emission lines of H I and He I are broad and have pronounced double peak profiles, consistent with the high orbital inclination shown by the eclipses. The continuum flux measured in June was steeper to blue and five times larger than that obtained in May. We do not classify this object as a mCV.

\subsubsection{CSS2200+03 (CSS100624:220031+033431)}

This object was discovered by CRTS and suggested to be a polar type $\mathrm{CV}$ in the CRTS circulars $^{8}$. Its light curve presents a quiescent level with long term (years) variability ranging from 18 to $19.5 \mathrm{mag}$ and several outbursts reaching about 16.5 mag. de Budè (2011) performed photometric follow-up observations, which yield non-eclipsing light curves (within a maximum time span of 3.6 h) dominated by flickering with less than $0.5 \mathrm{mag}$ amplitude.

Our exploratory spectrum displays broad Balmer and He I emission lines with double peak profiles, which are more conspicuous in the He I lines. The presence of a disk, evidenced by the double peak lines and corroborated by the outbursts, discards the polar classification. He II $4686 \AA$ and C III/N III are weak but present. The continuum shows absorption features, which could be associated to a cool secondary. The spectral features are somewhat similar to the spectrum of CTCV205630 . We classify it as a mCV candidate.

\subsubsection{RXS2223+07 (1RXS J222335.6+074515 $=U S N O-B 1.0$ 0977-0743560)}

Also selected as a new CV from the ROSAT catalog, 1RXS2223+07 presents a DN light curve with outbursts 2 mag above the average $19.5 \mathrm{mag}$ quiescent level (Denisenko \& Sokolovsky 2011). Our spectrum presents broad Balmer and He I emission lines, besides He II $4686 \AA$, on a slightly red continuum, consistent with a DN spectrum.

\subsubsection{SSS2242-66 (SSS110720:224200-662512 $=1 R X S$ J224150.4-662508)}

This source presents a CRTS light curve varying between 16.5 and 18 mag with two outbursts reaching 15-15.5 mag. Its position matches that of the X-ray source 1RXS J224150.4-662508. Our spectrum, obtained at OPD, shows emission lines of $\mathrm{H} \alpha, \mathrm{H} \beta, \mathrm{He}$ I and He II $4686 \AA$ over a continuum that is slightly steeper to the blue. The spectrum is very similar to the spectrum of the IP candidate CTCV2056-30 described above which, as in this case, also presents outbursts and X-ray

\footnotetext{
${ }^{8}$ http://nessi.cacr.caltech.edu/catalina/Circulars/ 1006241041184114393.html
} 
detection. These similarities may indicate an IP nature for SSS2242-66.

\subsubsection{CSS2319+33 (CSS111021:231909+331540)}

CSS2319+33 has a highly variable CRTS light curve with short and long timescales, ranging from 16.5 to $19.5 \mathrm{mag}$, but it does not show any outburst. It matches a blue source at SDSS DR8, with $u=17.45, g=17.79, r=17.68, i=17.36$ and $z=17.02$ and also matches the ROSAT source 1RXS J231909.9+331544. Despite being noisier, the OPD spectrum is very similar to the spectrum of SSS2242-66, which in turn resembles the spectrum of CTCV2056-30, so we consider it as an IP candidate.

\subsection{Systems with Disks in High Accretion State}

\subsubsection{RXS0721-05 (1RXS J072103.3-055854 $=$ USNO-B1.0 0840-0137592)}

Denisenko \& Sokolovsky (2011) identified this system as USNO-B1.0 0840-0137592, the optically variable counterpart of an X-ray source in the ROSAT catalog, and suggested a classification as a dwarf nova. Their analysis of archive photographic plates shows that most of the time the system is fainter than $17 \mathrm{mag}$ in the B band, but occasionally gets brighter, reaching $16.5 \mathrm{mag}$. Our exploratory spectrum has a flux in the $\mathrm{V}$ band of $\sim$ $15.3 \mathrm{mag}$, brighter than in any previous detection, corroborating a dwarf nova classification. Our spectrum is typical of a dwarf nova in eruption, showing narrow Balmer and He II $4686 \AA$ emission lines superimposed on a very blue continuum. During DN eruptions, broad absorption troughs may develop at the base of very narrow Balmer emission cores, mainly in the higher member of the Balmer series - see Warner (1995) and the case of SS Cyg by Hessman et al. (1984). These features are also observed in 1RXS0721-05. Emission lines of He I, the interstellar absorptions lines of Na I 5890 and $5896 \AA$, and the diffuse interstellar band (DIB) at $6284 \AA$ are also present in our spectrum. If we consider its ROSAT counts rate (Boller et al. 2016) and the quiescent optical magnitude, the object is located in the dwarf novae region in Figure 2 of Beuermann \& Thomas (1993). The ROSAT hardness ratios $(\mathrm{HR} 1=1.000 \pm 0.360$ and HR2 $=-0.108 \pm 0.276$ ) are also consistent with a dwarf nova classification (e.g., Hümmerich et al. 2014).

\subsubsection{SSS2042-60 (SSS110526:204247-604523)}

SSS2042-60 has a CRTS light curve with two distinct brightness levels at 18 and $19.5 \mathrm{mag}$, being observed most of the time at the higher state. No eruption was recorded. Our SOAR spectrum displays a very steep blue continuum with $\mathrm{H} \alpha$ in emission and $\mathrm{H} \beta$ in broad absorption with a weak central emission feature. He II $4686 \AA$ is marginally detected and $\mathrm{Na} \mathrm{I}$ absorption is visible. Our spectrum has a flux equivalent to $\mathrm{V}=18 \mathrm{mag}$, near the brightest state. We suggest the object is a novalike system, possibly of VY Scl type due to the fadings in its light curve.

\subsection{Nova}

\subsubsection{XMM0630-60 (XMMSL1 J063045.9-603110)}

This source was discovered by XMM-Newton in 2011 as a soft X-ray transient (2011 Dec 01) and was suggested to be a Nova for its X-ray softness (Read et al. 2011a). Observations with GROND identified the optical counterpart with g' $=18.4$ mag and $\mathrm{r}^{\prime}=19.5 \mathrm{mag}$, too faint for a recent Galactic Nova (Kann et al. 2011). The optical source has a blue SED, also atypical for a Nova, with a hint of strong He II emission. Swift observations show a very soft X-ray spectrum with $\mathrm{kT}$ $=48 \mathrm{eV}, 12$ times fainter than the XMM-Newton flux obtained 19 days before (Read et al. 2011b). XMM0630-60 was also selected as a blazar candidate, but this classification was not confirmed (Cowperthwaite et al. 2013).

Our spectrum is compatible with a Nova in the nebular phase and displays strong blended lines of [O III] 4959 and $5007 \AA$ A. Besides, broad and near rest position emission lines of He II $4686 \AA$, $\mathrm{H} \beta$ and $\mathrm{H} \alpha$ are also present, with multiple narrow components. Our spectrum corresponds to $\mathrm{V} \approx$ 21.8 mag.

\subsection{Miscellaneous Objects}

In this section we present objects initially considered as $\mathrm{mCV}$ candidates due to their CRTS light curves, showing variability that could be interpreted as, for example, distinct high/low states. Many of these candidates have also been 
pre-classified as $\mathrm{CV}$ candidates by CRTS. The exploratory spectra, however, have shown that they are not CVs, but actually can be classified as extragalactic objects - active galactic nuclei (AGN) which usually share observational features with CVs, like variability and color - or other types of variable stars as RR Lyrae or low and intermediate-mass pulsating red giants.

The first seven objects described below are classified as extragalactic sources because of the redshift of the lines identified in their spectra. Five of them are dominated by broad Mg II $2798 \AA$ lines characteristics of AGN. The remaining two objects display both emission and absorption features, with strengths typically observed in galaxies with on-going stellar formation. To the best of our knowledge, we provided here the first classification and redshifts for these sources. The next three objects are RR Lyrae stars and the last three are Mira or semi-regular (SR) pulsating red giants (Kholopov et al. 1985).

\subsubsection{CSS0213+00 (CSS091215:021311+002153)}

This CRTS object varies between 20.5 and 18.5 mag and was suggested to be a polar type CV or an $\mathrm{AGN}^{9}$. The exploratory spectrum is compatible with a Type I AGN at $\mathrm{z}=1.257$ based on the conspicuous broad emission line of Mg II $2798 \AA$ and numerous blended UV Fe II multiplets.

\subsubsection{CSS0315-01 (CSS071206:031525-014051)}

CSS0315-01 shows a CRTS light curve slowly varying between $\mathrm{V}=19$ and $\mathrm{V}=20.5 \mathrm{mag}$. A broad Mg II $2798 \AA$ line and the UV Fe II multiplets in the spectrum lead to a classification as a Type I AGN at $z=0.8513$, further confirmed by the detection of [Ne V] $3425 \AA$. [O II] $\lambda \lambda 3727,3729 \AA$ are also detected.

\subsubsection{CSS1240-15 (CSS071218:124027-150558)}

This CRTS transient is highly variable in the range from 18.5 to $21 \mathrm{mag}$. The spectrum presents narrow emission lines of [O II] 3726/3728 $\AA, \mathrm{H} \gamma$, $\mathrm{H} \beta$ and [O III] 4959/5007 $\AA$ at a redshift $z=$ 0.272 , besides absorption lines of $\mathrm{Ca} \mathrm{II} \mathrm{H}$ and $\mathrm{K}$. An upper limit to the flux ratio $[\mathrm{O} \mathrm{III}] / \mathrm{H} \beta$ is 0.39

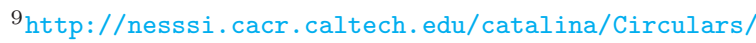
912151010124106434.html
}

, which classifies CSS1240-15 as a H II galaxy (Kewley et al. 2006), corroborated by the ratio $\left[\begin{array}{ll}\mathrm{O} & \mathrm{II}\end{array}\right] /\left[\begin{array}{ll}\mathrm{O} & \mathrm{III}\end{array}\right]=5.4 \pm 0.6$ (Baldwin et al. 1981). The large amplitude variability of this source, on the other hand, leads to a distinct classification as an AGN caught at a minimum state, with the central source completely hidden from direct view , or as a low excitation radio AGN (LERAG, Padovani et al. 2015). Follow-up spectroscopy is necessary to unveil the real nature of this object.

\subsubsection{MLS1251-02 (MLS110329:125118-020208)}

This source is highly variable in CRTS light curve, ranging from 16 to 22 mag in timescales of months. The exploratory spectrum corresponds to an AGN at $\mathrm{z}=1.1887$, determined from a emission line at $6120 \AA$, which we identify as Mg II $2798 \AA$. Absorption doublets are clearly visible at $5700 \AA$ and $5010 \AA$. These are Mg II $2796,2803 \AA$ absorption-line systems from two distinct intervening gaseous clouds in the line of sight of the AGN, at redshifts of $\mathrm{z}_{1}=1.037$ and $\mathrm{z}_{2}=0.791$, respectively. Absorption features like the doublet of Fe II 2586,2600 $⿱$, visible at $4640 \AA$, and the $\mathrm{Mg}$ I $2852 \AA$ line, visible at $5108 \AA$, are also present and are associated to the gaseous structure at $\mathrm{z}_{2}=0.791$. The strong variability of MLS125102 suggests that it is an optically violently variable (OVV) quasar.

\subsubsection{MLS1402-09 (MLS110301:140203-090329)}

The CRTS light curve of MLS1402-09 displays a low brightness level at 20.5 average magnitude and a high level at $19.5-20 \mathrm{mag}$. The spectrum shows a prominent narrow emission line at 5493 $\AA$, which we identify as [O II] $3726 / 3728 \AA$ and from which we derived $z=0.4736$, and absorption lines of $\mathrm{Ca}$ II $\mathrm{H}$ and $\mathrm{K}$. In the NVSS catalog there is a radio source (NVSS J140203-090747) offset by $4.307^{\prime}$ from MLS1402-09 with a measured flux of $3.2 \pm 0.6 \mathrm{mJy}$ at $1.4 \mathrm{GHz}$. This flux level classifies it as a low-emission radio AGN (LERAG, Padovani et al. 2015).

\subsubsection{MLS1404-15 (MLS100617:140447-152226)}

MLS1404-15 was suggested to be a CV by CRTS from its blue DSS color and high variability. The CRTS light curve has a low state at 19.5 average magnitude, changing to a high state at av- 
erage 18.5 mag from 2009 on. A prominent broad feature centered at $5321 \AA$, that we associated to Mg II $2798 \AA$ at $z=0.9017$, dominates the spectrum. We also identified the UV Fe II multiplets at both sides of Mg II 2798, typical of Type I AGNs. The presence of high-ionization lines of [Ne V] 3347,3427 $\AA$ and the permitted O III line at $3134 \AA$ give additional support that MLS140415 is indeed an AGN.

\subsubsection{MLS2230-04 (MLS100906:223034-042347)}

Classified as a possible variable star by CRTS, MLS2230-04 has a bimodal light curve with a low state at $20 \mathrm{mag}$ and a high state at $19.3 \mathrm{mag}$. The SOAR spectrum is dominated by a broad Mg II $2798 \AA$ emission line and hints of UV Fe II multiples at both sides of $\mathrm{Mg}$ II, classifying this source as a Type I AGN at $\mathrm{z}=0.968$.

\subsubsection{The RR Lyrae Stars MLS1519-13, MLS2044- 16 and MLS2132-15}

MLS1519-13, MLS2044-16 and MLS2132-15 were selected for their CRTS light curve variability. The identification spectra are consistent with A or F spectral type, with narrow Balmer absorption lines over a blue continuum. The time-series analysis of the CRTS photometric data yielded periods of $0.521 \mathrm{~d}$ for MLS1519-13, $0.535 \mathrm{~d}$ for MLS2044-16 and 0.486 d for MLS2132-15. The CRTS light curves of these RR Lyrae stars, folded on the respective periods, are shown in Figure 5.

\subsubsection{The Pulsating Red Giants Stars SSS1448- 40, SSS1639-23 and SSS1940-63}

SSS1448-40, SSS1639-23 and SSS1940-63 were also selected from the CRTS variability. The exploratory and fitted reference spectra for spectral type estimation are presented in Figure 6. The DFT and Lomb-Scargle period search methods applied to the CRTS data yielded a period of $87 \pm 1$ d for SSS1448-40 and $307 \pm 16$ d for SSS1639-23, but no coherent period was obtained for SSS194063 (Figure 7).

\section{SUMMARY AND DISCUSSION}

We report on an exploratory spectroscopy survey of magnetic CVs based on objects selected

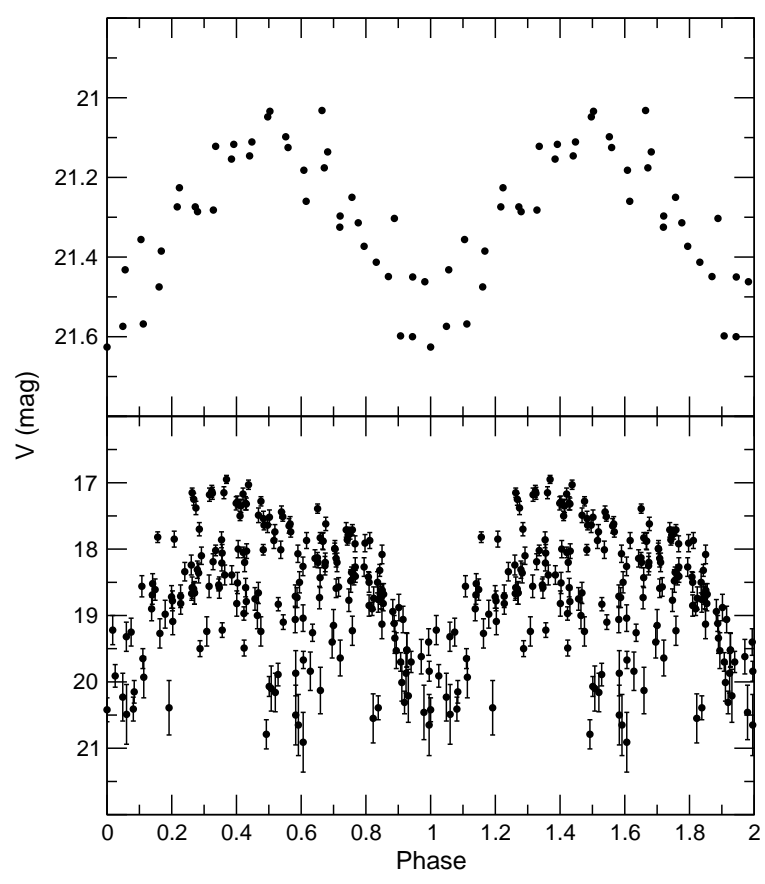

Fig. 4.- OPD (upper panel) and CRTS (lower nanel) lioht curves of MTS1609-10 hoth folded

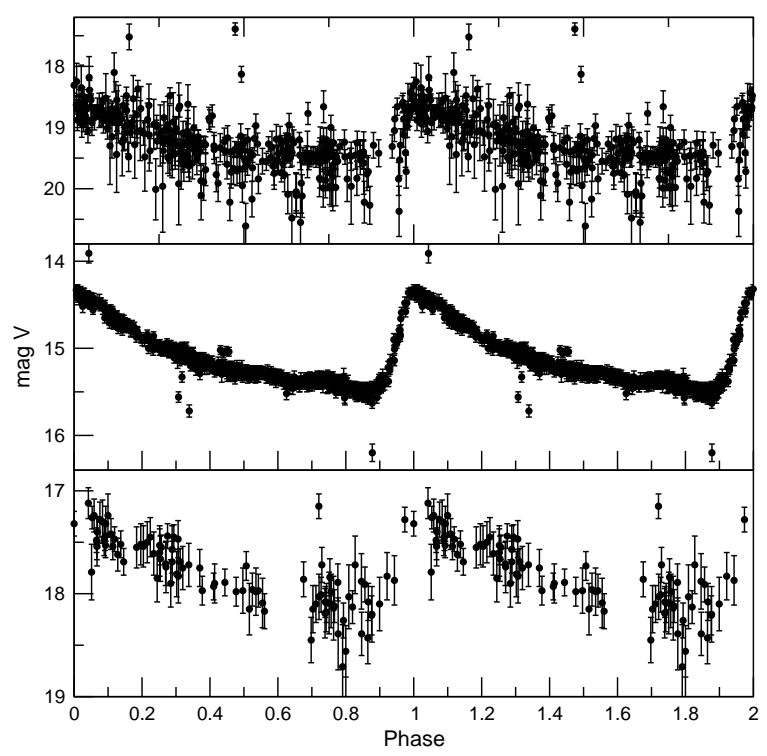

Fig. 5.- CRTS photometric data of MLS151913 (top), MLS2044-16 (middle) and MLS2132-15 (bottom) folded with the periods described in the text. 
mainly from the CRTS photometric transient survey but also from the literature. The main purpose of this work is to improve the statistics of the mCV class. From an initial sample of 45 objects, we found 32 sources which we classified as CVs based on the aspect of the exploratory spectra. This group is composed of 13 polar candidates, 9 IP candidates, 7 DNs in quiescence, 2 objects in high-accretion state and 1 nova. In addition to CVs, we have also found RR Lyrae stars, variable red giants and extragalactic objects. These results are summarized below.

From the group of 13 polar candidates, 5 are new classifications (MLS0456+18, MLS0720+17, MLS0854+13, CSS1127-05 and MLS1609-10) and other 6 are already suggested polars for which we provide the first spectrum and confirm the classification. The remaining 2 polar candidates present notable spectral variability relative to published spectra. In the Ritter \& Kolb (2003) catalog (update RKcat7.23, 2015), there are 119 confirmed and 30 uncertain polars. Six objects of our sample are not included in RKcat7.23. Only 6 objects (out of 13 polar candidates) are known highenergy sources, which shows that the X-rays detection is not a sine qua non criterion for the classification as a polar (or as a magnetic CV, see also Margon et al. (2014)). This is particularly true for faint/distant objects, which could be missed by soft X-rays surveys - see, for instance, the discussion of MLS0227+13 presented in Silva et al. (2015). Ten objects obey the two Silber's criteria for mCV classification (Silber 1992), hence Silber's criteria seems to be adequate to define polars. The three remaining objects are peculiar in some way: 1RXS1002-19, which has emission lines too broad for a polar; CSS1127-05, which has the largest $\mathrm{H} \beta$ EW of our CV sample; and CSS1503-22, which presents absorption features in the $\mathrm{H} \beta$ region preventing a proper quantification of line indicators. We present the first estimates for the orbital periods of MLS0456+18 ( $\mathrm{P}=2.36 \mathrm{~h})$ and MLS1609-10 $(\mathrm{P}=1.85 \mathrm{~h})$.

We classify 16 objects as probable $\mathrm{CV}$ with quiescent disks, including 1 confirmed IP and 8 possible IPs. CTCV2056-30 is a strong IP candidate due to its photometric variability modulated by the white dwarf rotation (Augusteijn et al. 2010). Most of the IP candidates present the He II $4686 \AA$ line, although in low intensity.
CSS0104-03, however, has this line strong enough to have its EW measured and, even in this case, its $\mathrm{EW}$ is smaller than $40 \%$ of $\mathrm{H} \beta \mathrm{EW}$, therefore too weak to obey the Silber's criteria for a mCV. Even so, we classify it as a probable IP because its He II $4686 \AA$ line is stronger in quiescence compared to the brighter state, contrary to what is expected from non-magnetic CVs. The 7 remaining new IP candidates (namely CSS1012-18, SSS1359-39, MLS2043-19, MLS205419, CSS2200+03, SSS2242-66 and CSS2319+33) were classified as such by spectral similarity to CTCV2056-30. All our nine IP candidates have He II $4686 \AA$ in the spectra - but none obeys Silber's criteria - 4 of them have X-ray counterparts and 8 have shown outbursts.

The spectrum of CTCV2056-30, whose orbital period is around $1.75 \mathrm{~h}$, is very similar to the spectrum of several short-period IPs, like SDSS J2333, HT Cam and DW Cnc. Could our IP candidates spectrally similar to CTCV2056-30 have short periods? While most of the polars have orbital periods below $4 \mathrm{~h}$, few IPs have periods below the 2-3 h period gap. The Ritter \& Kolb (2003) cata$\log$ registers 68 confirmed IPs and only 9 of those have orbital periods below the gap. This was interpreted as an evolution of long-period/high-B IPs to short period polars, whereas those with lower magnetic fields remain as IPs (Norton et al. 2004). This kind of conclusion demands a sample of short period IPs as complete as possible. Follow-up studies of these candidates are essential, and may confirm their nature and help to build an homogeneous sample of IPs, which can be used to investigate the issue of $\mathrm{mCV}$ evolution.

Ten remaining objects from our sample are CVs as well. Seven are DNs with quiescent disks and two have disks in high state of accretion: the known dwarf nova 1RXS0721-05, which we caught in eruption, and the newly discovered CV SSS2042-60, which we classify as a novalike variable, possibly of VY Scl type. We also confirmed that the transient high-energy source XMM063060 is a nova, which we observed in the nebular phase.

Previous similar works (e.g., Drake et al. 2014; Szkody et al. 2011, 2014; Thorstensen \& Skinner 2012; Thorstensen et al. 2016) targeted on the discovery of CVs from variability or color-based selected samples, have indirectly shown that the 
identification of a $\mathrm{mCV}$ is not a trivial task. Usually, no isolated observational technique is enough to confirm the classification as a $\mathrm{mCV}$, specially in the case of IPs. The usual criteria include X-ray detection, the relative intensity of the He II $4686 \AA$ emission line and polarized optical/IR emission. But none of these criteria is essential to the $\mathrm{mCV}$ classification, while some are not exclusive of the $\mathrm{mCV}$ class. A mCV may not be detected as an Xray source and, on the other hand, many DNs are $\mathrm{X}$-ray emitters. The high-ionization line of He II $4686 \AA$ is present, for instance, in non-magnetic $\mathrm{CVs}$ like post-novae, novalike systems and close binary supersoft X-ray sources (CBSS, Kahabka 2006; Oliveira \& Steiner 2004) and, with lower intensity, in DNs as well. Also, few IPs have polarized emission. However, variability-based selection followed by spectroscopic snapshot observations of the selected targets may be a first step for the discovery of $\mathrm{mCVs}$, being a relatively inexpensive strategy in terms of telescope time. More than $70 \%$ of our sample are CVs and almost $50 \%$ of the sample was classified as mCVs. If the classifications from this work are confirmed, it would represent an increase of about $4 \%$ in the number of known polars and $12 \%$ in the number of known IPs. The confirmation of the classifications and the detailed nature of the CVs will be determined by time-resolved spectroscopic, photometric and polarimetric follow-up observations in the future.

We would like to thank the anonymous referee for useful comments, which helped to improve the paper. We also thanks J. E. Steiner and M. P. Diaz for important suggestions on the classification of some objects. This study was partially supported by CNPq (CVR: 306701/2015-4 and 306103/2012-5, KMGS:302071/2013-0, ARA: 311935/2015-0), FAPESP (LAA: 2012/097166 and 2013/18245-0; CVR: 2013/26258-4) and CAPES (MSP: 23038.009634/2016-71). This work is based partly on observations made at the Observatório do Pico dos Dias, Brazil, operated by the Laboratório Nacional de Astrofísica, and at the Southern Astrophysical Research (SOAR) telescope, which is a joint project of the Ministério da Ciência, Tecnologia e Inovação (MCTI) da República Federativa do Brasil, the US National Optical Astronomy Observatory (NOAO), the University of North Carolina at Chapel Hill (UNC) and Michigan State University (MSU). The CRTS survey is supported by the US National Science Foundation under grants AST-0909182. The CSS survey is funded by the National Aeronautics and Space Administration under grant no. NNG05GF22G issued through the Science Mission Directorate Near-Earth Objects Observations Program. STSDAS is a product of the Space Telescope Science Institute, which is operated by AURA for NASA.

Facilities: SOAR (Goodman HTS), LNA:1.6m Software: IRAF

\section{REFERENCES}

Afanasiev, V. L., Borisov, N. V., \& Gabdeev, M. M. 2015, AstBu, 70, 328

Augusteijn, T., Tappert, C., Dall, T., \& Maza, J. 2010, MNRAS, 405, 621

Baldwin, J. A., Phillips, M. M., \& Terlevich, R. 1981, PASP, 93, 5

Beuermann, K., \& Schwope, A. D. 1994, ASPC 56, Interacting Binary Stars, ed. A.W. Shafter (San Francisco, CA: ASP), 119

Beuermann, K., \& Thomas, H.-C. 1993, AdSpR, 13,115

Boller, T., Freyberg, M. J., Trümper, J., et al. 2016, A\&A, 588, A103

Breedt, E., Gänsicke, B. T., Drake, A. J., et al. 2014, MNRAS, 443, 3174

Clarke, D. 2002, A\&A, 386, 763

Clemens, J. C., Crain, J. A., \& Anderson, R. 2004, Proc. SPIE, 5492, 331

Coppejans, D. L., Körding, E. G., Knigge, C., et al. 2016, MNRAS, 456, 4441

Coppejans, D. L., Woudt, P. A., Warner, B., et al. 2014, MNRAS, 437, 510

Cowperthwaite, P. S., Massaro, F., D'Abrusco, R., et al. 2013, AJ, 146, 110 
Costa, J. E. R., \& Rodrigues, C. V. 2009, MNRAS, 398, 240

Cropper, M. 1990, SSRv, 54, 195

de Budè, D. L. 2011, Master of Science Thesis (Univ. of Cape Town)

Deeming, T. J. 1975, Ap\&SS, 36, 137

Denisenko, D., \& Martinelli, F. 2012, PZ, 32, 3

Denisenko, D. V., \& Sokolovsky, K. V. 2011, AstL, 37,91

Downes, R. A., Webbink, R. F., Shara, M. M., et al. 2001, PASP, 113, 764

Drake, A. J., Djorgovski, S. G., Mahabal, A., et al. 2009, ApJ, 696, 870

Drake, A. J., Gänsicke, B. T., Djorgovski, S. G., et al. 2014, MNRAS, 441, 1186

Drissen, L., Shara, M. M., Dopita, M., \& Wickramasinghe, D. T. 1994, AJ, 107, 2172

Dworetsky, M. M. 1983, MNRAS, 203, 917

Ferrario, L., de Martino, D., \& Gänsicke, B. T. 2015, Space Sci. Rev., 191, 111

Fertig, D., Mukai, K., Nelson, T., \& Cannizzo, J. K. 2011, PASP, 123, 1054

Gray, R. O., \& Corbally, C., J. 2009, Stellar Spectral Classification (Princeton, NJ: Princeton Univ. Press)

Gunn, J. E., \& Stryker, L. L. 1983, ApJS, 52, 121

Hamuy, M., Walker, A. R., Suntzeff, N. B., et al. 1992, PASP, 104, 533

Hardy, L. K, McAllister, M. J, Dhillon, V. S, et al. 2016, arXiv:1611.07885

Hessman, F. V., Robinson, E. L., Nather, R. E., \& Zhang, E.-H. 1984, ApJ, 286, 747

Horne, J. H., \& Baliunas, S. L. 1986, ApJ, 302, 757

Hümmerich, S., Bernhard, K., \& Srdoc, G. 2014, OEJV 167, http://var.astro.cz/ oejv/oejv $\cdot$ php?lang=en
Kahabka, P. 2006, Advances in Space Research, 38,2836

Kann, D. A., Greiner, J., \& Rau, A. 2011, ATel. 3813, http://www.astronomerstelegram. org/?read=3813

Kato, T., Hambsch, F.-J., Maehara, H., et al. 2013, PASJ, 65, 23

Kemp, J., Patterson, J., Thorstensen, J. R., et al. 2002, PASP, 114, 623

Kewley, L. J., Groves, B., Kauffmann, G., \& Heckman, T. 2006, MNRAS, 372, 961

Kholopov, P. N., Samus, N. N., Durlevich, O. V., et al. 1992, General Catalogue of Variable Stars, 4rd ed., vol.IV, Bull. Inf. CDS, 40, 15

Knigge, C., Baraffe, I., \& Patterson, J. 2011, ApJS, 194, 28

Kovacs, G. 1981, Ap\&SS, 78, 175

Kryachko, T., Samokhvalov, A., \& Satovskiy, B. 2010, IBVS 5952, http://www.konkoly.hu/ cgi-bin/IBVS?5952

Külebi, B., Jordan, S., Euchner, F., Gänsicke, B. T., \& Hirsch, H. 2009, A\&A, 506, 1341

Levitan, D., Groot, P. J., Prince, T. A., et al. 2015, MNRAS, 446, 391

Lomb, N. R. 1976, Ap\&SS, 39, 447

Margon, B., Levitan, D., Prince, T. A., Hallinan, G., \& PTF Collaboration 2014, Stellar Novae: Past and Future Decades, 490, 389

Mennickent, R. E., Tovmassian, G., Zharikov, S. V., et al. 2002, A\&A, 383, 933

Mortier, A., Faria, J. P., Correia, C. M., Santerne, A., \& Santos, N. C. 2015, A\&A, 573, A101

Mróz, P., Pietrukowicz, P., Poleski, R., et al. 2013, Acta Astron., 63, 135

Norton, A. J., Wynn, G. A., \& Somerscales, R. V. 2004, ApJ, 614, 349

Oliveira, A. S., \& Steiner, J. E. 2004, MNRAS, 351,685 
Ohshima, T. 2013, vsnet-alert 16294, http://ooruri.kusastro.kyoto-u.ac. $\mathrm{jp} / \mathrm{mailarchive/vsnet-alert/16294}$

Padovani, P., Bonzini, M., Kellermann, K. I., Miller, N., Mainieri, V., Tozzi, P. 2015, MNRAS, 452, 1263

Patterson, J., Thorstensen, J. R., Vanmunster, T., et al. 2004, PASP, 116, 516

Pojmanski, G. 2002, AcA, 52, 397

Pretorius, M. L., Knigge, C., \& Schwope, A. D. 2013, MNRAS, 432, 570

Ramsay, G., \& Cropper, M. 2003, MNRAS, 338, 219

Read, A. M., Saxton, R. D., \& Esquej, P. 2011a, ATel. 3811, http://www. astronomerstelegram.org/?read=3811

Read, A. M., Saxton, R. D., \& Esquej, P. 2011b, ATel. 3821, http://www. astronomerstelegram.org/?read=3821

Ritter, H., \& Kolb, U. 2003, A\&A, 404, 301 (update RKcat7.23, 2015)

Rodrigues, C. V., Jablonski, F. J., D'Amico, F., et al. 2006, MNRAS, 369, 1972

Rodrigues, C. V., Taylor, K., Jablonski, F. J., et al. 2012, Proc. SPIE, 8446, 844626

Rodríguez-Gil, P., Gänsicke, B. T., AraujoBetancor, S., \& Casares, J. 2004, MNRAS, 349, 367

Saxton, R. D., Read, A. M., Esquej, P., et al. 2008, A\&A, 480, 611

Scargle, J. D. 1982, ApJ, 263, 835

Schmidt, G. D., Szkody, P., Vanlandingham, K. M., et al. 2005, ApJ, 630, 1037

Schwope, A. D., \& Thinius, B. 2012, AN, 333, 717

Silber, A. D. 1992, Ph.D. Thesis (MIT)

Silva, K. M. G., Rodrigues, C. V., Costa, J. E. R., et al. 2013, MNRAS, 432, 1587
Silva, K. M. G., Rodrigues, C. V., Oliveira, A. S., et al. 2015, MNRAS, 451, 4183

Southworth, J., Gänsicke, B. T., Marsh, T. R., de Martino, D., \& Aungwerojwit, A. 2007, MNRAS, 378, 635

Stellingwerf, R. F. 1978, ApJ, 224, 953

Szkody, P., Anderson, S. F., Agüeros, M., et al. 2002, AJ, 123, 430

Szkody, P., Fraser, O., Silvestri, N., et al. 2003, AJ, 126, 1499

Szkody, P., Henden, A., Fraser, O., et al. 2004, AJ, 128, 1882

Szkody, P., Henden, A., Agüeros, M., et al. 2006, AJ, 131, 973

Szkody, P., Henden, A., Mannikko, L., et al. 2007, AJ, 134, 185

Szkody, P., Anderson, S. F., Hayden, M., et al. 2009, AJ, 137, 4011

Szkody, P., Anderson, S. F., Brooks, K., et al. 2011, AJ, 142, 181

Szkody, P., Everett, M. E., Howell, S. B., et al. 2014, AJ, 148, 63

Szkody, P., Henden, A., Fraser, O. J., et al. 2005, AJ, 129, 2386

Thomas, H.-C., Beuermann, K., Reinsch, K., Schwope, A. D., \& Burwitz, V. 2012, A\&A, 546, A104

Thorstensen, J. R., \& Skinner, J. N. 2012, AJ, 144,81

Thorstensen, J. R., Alper, E. H., \& Weil, K. E. 2016, arXiv:1609.02215

Warner, B. 1995, Cataclysmic Variable Stars (1st ed.; Cambridge, GB: CUP)

Woudt, P. A., Warner, B., de Budé, D., et al. 2012, MNRAS, 421, 2414

York, D. G., Adelman, J., Anderson, J. E., Jr., et al. 2000, AJ, 120, 1579 
Zacharias, N., Monet, D. G., Levine, S. E., et al. 2004, BAAS, 36, 1418

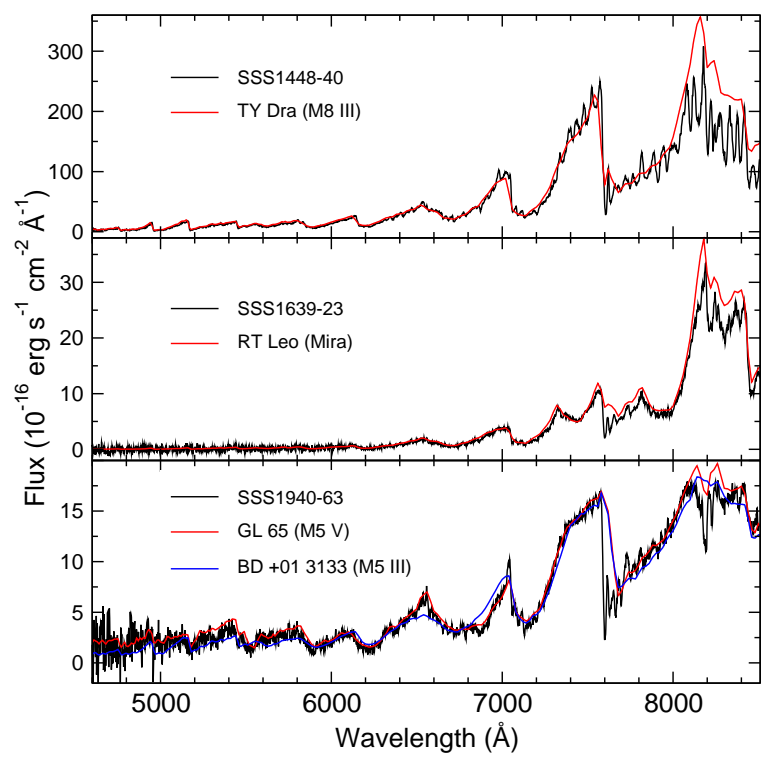

Fig. 6.- Spectra of SSS1448-40 (top), SSS163923 (middle) and SSS1940-63 (bottom). The black lines are our spectra and the red and blue lines are the fitted reference spectra used for the spectral type estimation, from the Bruzual-Persson-GunnStryker Atlas (Gunn \& Stryker 1983).

This 2-column preprint was prepared with the AAS LATEX macros v5.2. 


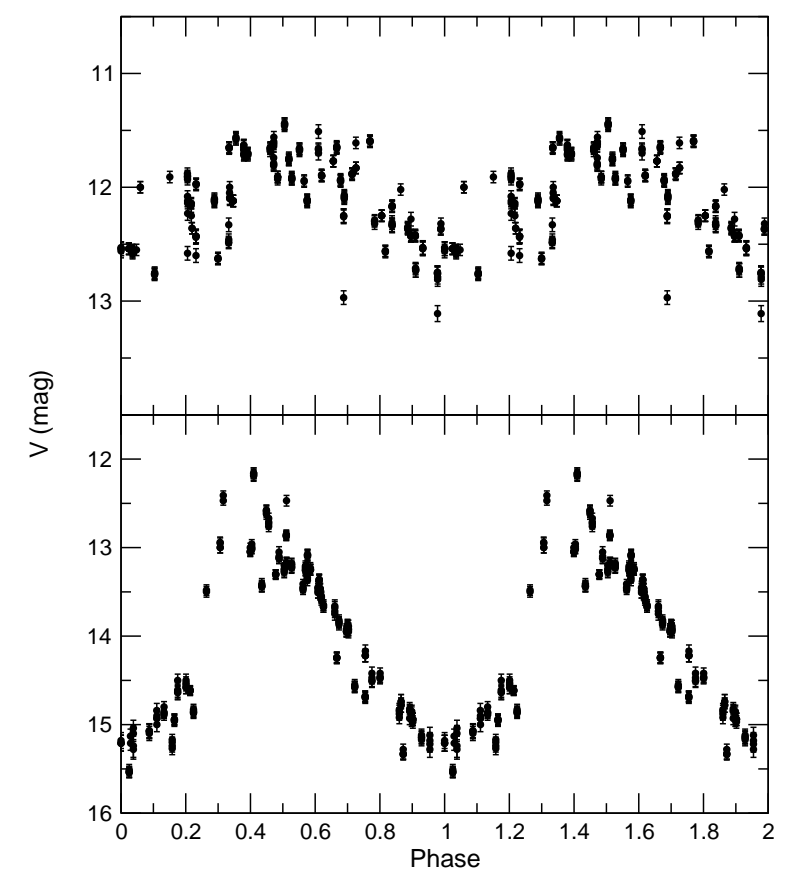

Fig. 7.- CRTS photometric light curves of SSS1448-40 (top) and SSS1639-23 (bottom) folded with periods of $87 \mathrm{~d}$ and $307 \mathrm{~d}$, respectively - see text. 